\title{
Orbital Structure of Merger Remnants. I. Effect of Gas Fraction in Pure Disk Mergers
}

\section{Citation}

Hoffman, Loren, Thomas J. Cox, Suvendra Dutta, and Lars Hernquist. 2010. “ORBITAL STRUCTURE OF MERGER REMNANTS. I. EFFECT OF GAS FRACTION IN PURE DISK MERGERS." The Astrophysical Journal 723 (1): 818-44. https://doi.org/10.1088/0004-637x/723/1/818.

\section{Permanent link}

http://nrs.harvard.edu/urn-3:HUL.InstRepos:41381852

\section{Terms of Use}

This article was downloaded from Harvard University's DASH repository, and is made available under the terms and conditions applicable to Other Posted Material, as set forth at http:// nrs.harvard.edu/urn-3:HUL.InstRepos:dash.current.terms-of-use\#LAA

\section{Share Your Story}

The Harvard community has made this article openly available.

Please share how this access benefits you. Submit a story.

Accessibility 


\title{
ORBITAL STRUCTURE OF MERGER REMNANTS. I. EFFECT OF GAS FRACTION IN PURE DISK MERGERS
}

\author{
Loren Hoffman $^{1,2}$, Thomas J. Cox ${ }^{3,4}$, Suvendra Dutta $^{3,5}$, and Lars Hernquist ${ }^{3}$ \\ ${ }^{1}$ Department of Physics and Astronomy, Northwestern University, Dearborn Observatory, 2131 Tech Drive, Evanston, IL 60208, USA; loren@phys.huji.ac.il \\ ${ }^{2}$ Racah Institute of Physics, The Hebrew University, Jerusalem 91904, Israel \\ ${ }^{3}$ Department of Astronomy, Harvard University, 60 Garden Street, Cambridge, MA 02138, USA \\ ${ }^{4}$ Carnegie Observatories, 813 Santa Barbara Street, Pasadena, CA 91101, USA \\ ${ }^{5}$ Faculty of Arts and Sciences, Harvard University, University Hall, Cambridge, MA 02138, USA \\ Received 2009 December 28; accepted 2010 September 6; published 2010 October 15
}

\begin{abstract}
Since the violent relaxation in hierarchical merging is incomplete, elliptical galaxies retain a wealth of information about their formation pathways in their present-day orbital structure. Recent advances in integral field spectroscopy, multi-slit infrared spectroscopy, and triaxial dynamical modeling techniques have greatly improved our ability to harvest this information. A variety of observational and theoretical evidence indicates that gas-rich major mergers play an important role in the formation of elliptical galaxies. We simulate 1:1 disk mergers at seven different initial gas fractions $\left(f_{\text {gas }}\right)$ ranging from $0 \%$ to $40 \%$, using a version of the TreeSPH code Gadget- 2 that includes radiative heating and cooling, star formation, and feedback from supernovae and active galactic nuclei. We classify the stellar orbits in each remnant and construct radial profiles of the orbital content, intrinsic shape, and orientation. The dissipationless remnants are typically prolate-triaxial, dominated by box orbits within $r_{c} \sim 1.5 R_{e}$, and by tube orbits in their outer parts. As $f_{\text {gas }}$ increases, the box orbits within $r_{c}$ are increasingly replaced by a population of short-axis tubes ( $z$-tubes) with near zero net rotation, and the remnants become progressively more oblate and round. The long-axis tube ( $x$-tube) orbits are highly streaming and relatively insensitive to $f_{\text {gas }}$, implying that their angular momentum is retained from the dynamically cold initial conditions. Outside $r_{c}$, the orbital structure is essentially unchanged by the gas. For $f_{\text {gas }} \gtrsim 15 \%$, gas that retains its angular momentum during the merger re-forms a disk that appears in the remnants as a highly streaming $z$-tube population superimposed on the hot $z$-tube distribution formed by the old stars. In the 15\%-20\% gas remnants, this population appears as a kinematically distinct core (KDC) within a system that is slowly rotating or dominated by minor-axis rotation. These remnants show an interesting resemblance, in both their velocity maps and intrinsic orbital structure, to the KDC galaxy NGC 4365. At 30\%-40\% gas, the remnants are rapidly rotating, with sharp embedded disks on $\sim 1 R_{e}$ scales. We predict a characteristic, physically intuitive orbital structure for 1:1 disk merger remnants, with a distinct transition between 1 and $3 R_{e}$ that will be readily observable with combined data from the two-dimensional kinematics surveys SAURON and SMEAGOL. Our results illustrate the power of direct comparisons between $N$-body simulations and dynamical models of observed systems to constrain theories of galaxy formation.
\end{abstract}

Key words: galaxies: elliptical and lenticular, $\mathrm{cD}$ - galaxies: formation - galaxies: interactions - galaxies: kinematics and dynamics - galaxies: structure - methods: numerical

Online-only material: color figures

\section{INTRODUCTION}

\subsection{Motivation}

In the standard $\Lambda \mathrm{CDM}$ concordance cosmology (Ostriker \& Steinhardt 1995; Dodelson et al. 1996; Spergel et al. 2007), structure in the universe grows hierarchically, through a progression of smaller bodies accreting material and merging to form larger systems (e.g., White \& Rees 1978). Cosmological $\mathrm{N}$-body simulations starting from a Gaussian random field of linear density fluctuations at high redshift (e.g., Springel et al. 2005b), together with analytic models of gravitational collapse (e.g., Bertschinger 1985), have given us a fairly clear picture of how dark matter (DM) assembles in the universe. Extended Press-Schechter theory (Press \& Schechter 1974; Bond et al. 1991; Lacey \& Cole 1993) provides an analytic formalism for computing halo merger rates and assembly histories, which matches $N$-body simulations remarkably well (Lacey \& Cole 1994; Genel et al. 2009a), and has been used in a variety of semianalytic models of structure formation (e.g., Cole et al. 2000; Manrique \& Salvador-Sole 1996; Kauffmann et al. 1999; Somerville \& Primack 1999; Croton et al. 2006; Somerville et al. 2008; Stewart et al. 2009a). Simulated DM halos also appear to share a universal internal morphology, with density and velocity anisotropy profiles similar to the generic outcome of violent relaxation following dissipationless collapse or strong tidal shocking (Dubinski \& Carlberg 1991; Navarro et al. 1997; Bullock et al. 2001a, 2001b; Navarro et al. 2010; Miller \& Smith 1979; van Albada 1982; McGlynn 1984, 1990; Spergel \& Hernquist 1992; Huss et al. 1999; MacMillan et al. 2006; Bellovary et al. 2008).

There is no such simple theory of hierarchical galaxy formation, because the luminous components of galaxies are formed through complex baryonic physics. For instance radiative heating and cooling, star formation (SF) and gas expulsion through stellar winds, energy, and momentum feedback from supernovae and active galactic nuclei (AGNs), ram pressure stripping, and resonant effects in dynamically cold systems, are all important ingredients in determining the baryonic structure of galaxies (e.g., Croton et al. 2006; Springel 2000; Springel et al. 2005a; Best et al. 2007; Cox et al. 2006b; Ceverino \& Klypin 2009; Martig \& Bournaud 2009; D'Onghia et al. 2009). Cosmological models of galaxy formation must therefore be calibrated with high-resolution simulations on galactic scales that study 
these effects in isolation, coupled with direct constraints from observations.

Violent relaxation in mergers tends to drive galaxies toward a universal, fully mixed structure (Alladin 1965; Lynden-Bell 1967; Syer \& White 1998; Thomas et al. 2009), while gas accretion and stellar outflows produce new dynamically cold components (Rix \& White 1990; Block et al. 2002; Genel et al. 2009b; Dekel et al. 2009; Bournaud \& Elmegreen 2009; Martig \& Bournaud 2009; Daddi et al. 2010). From the hot stellar distributions of elliptical galaxies, we deduce that they are the most merger-dominated systems. Ellipticals and bulges contain the majority of the stellar mass in the local universe (e.g., Gadotti 2009), and often serve as a testing ground for theory since they are the most evolved under the complex combination of processes driving galaxy formation. Since the violent relaxation in mergers is incomplete, elliptical galaxies retain a wealth of information about their formation histories in their presentday distribution functions (e.g., Eggen et al. 1962; Lynden-Bell 1967; White 1980; Valluri et al. 2007).

Recent advances in integral field spectroscopy (IFS; Bacon et al. 1995, 2001; Hill et al. 2006; Weijmans et al. 2009), multislit infrared spectroscopy (Norris et al. 2008; Proctor et al. 2009), and triaxial dynamical modeling techniques (van de Ven et al. 2008; van den Bosch et al. 2008; de Lorenzi et al. 2006, 2009) have greatly improved our ability to harvest this information. The SAURON project (Bacon et al. 2001; Emsellem et al. 2004 ) will produce high resolution, two-dimensional kinematic maps within $\sim 1$ effective radius $\left(R_{e}\right)$ for a representative sample of $\sim 100$ nearby elliptical galaxies and spiral bulges, using a panoramic integral field spectrograph mounted on the William Herschel Telescope. The data on 48 early-type galaxies released to date have revealed an unexpectedly rich variety of kinematic structures, which poses a new challenge for galaxy formation simulations (Jesseit et al. 2007; Burkert et al. 2008). Dynamical modeling studies have shown that, in practice, two-dimensional maps of the first four moments $\left(h_{1}-h_{4}\right)$ of the line-of-sight velocity distribution (LOSVD) provided by SAURON are typically sufficient to uniquely reconstruct the three-dimnesional stellar orbital distribution (van de Ven et al. 2008; van den Bosch et al. 2008). Complex features present in many systems, such as embedded disks and kinematically distinct cores (KDCs), can provide especially strong constraints on the intrinsic structure (van den Bosch \& van de Ven 2008).

A good example of the power of these new techniques is provided by the case of NGC 4365 . This massive old elliptical is known for its minor axis rotation (Wagner et al. 1988; Bender et al. 1994) and KDC (Davies et al. 2001), and is therefore a natural candidate for dynamical modeling. Statler et al. (2004) modeled this galaxy using a velocity field-fitting method (Statler 1994a, 1994b) that made use of the surface brightness and full two-dimensioanl velocity map from SAURON, but not the higher moments of the LOSVDs. They found that the system was nearly maximally triaxial, ruling out axisymmetry at $>95 \%$ confidence.

A few years later, van den Bosch et al. (2008) modeled the same galaxy using an advanced new triaxial Schwarzschild modeling (Schwarzschild 1979) code that can incorporate all of the LOSVD moments up to $h_{4}$. They reached a qualitatively different conclusion-that the system was nearly oblate axisymmetric. The predominance of the minor-axis rotation in the outer parts of the map owed to a high degree of cancellation of the orbits rotating in a prograde and retrograde sense about the short axis, and streaming of the smaller population of orbits rotating about the intrinsic long axis. They found no major transition in the orbital structure at the boundary of the kinematically "decoupled" core, making it unlikely that it formed in a separate infall event. The orbital structure of the 15\%-20\% gas merger remnants in this paper bears a tantalizing resemblance to that of NGC 4365 (cf. e.g., our Figures 9-10 and 17-18 with Figures 7, 11, and 12 of van den Bosch et al. 2008).

However, a limitation of the SAURON spectrograph is its small field of view, corresponding to $\sim 1 R_{e}$ on a typical elliptical target. The outer parts of galaxies are less relaxed than their inner parts, and bar-like modes in mergers efficiently transport angular momentum outward (Ostriker \& Peebles 1973; Fall 1979; Hernquist 1993; Barnes \& Hernquist 1996; Hopkins et al. 2008c; L. Hoffman et al. 2009b, in preparation). A gasrich merger between two spiral galaxies with halos might be expected to produce a remnant with three distinct components in its distribution function: (1) an inner part formed through dissipation, (2) a middle part reflecting the dynamically cold distribution of the disk stars, and (3) an outer part arising from the pre-existing stellar halo populations. Observations with about four times the spatial coverage of SAURON would be able to detect these dynamical subcomponents and probe the parts of galaxies retaining the most memory of their progenitors' angular momentum and internal structure. Hints of increased complexity in the angular momentum profiles at large radii have indeed been observed in a few elliptical galaxies (Coccato et al. 2009; Proctor et al. 2009).

A number of projects designed to extend SAURON-style dynamical modeling out to larger radii is currently underway. The SMEAGOL survey (Proctor et al. 2009; Foster et al. 2009) will obtain smoothed two-dimensional maps of $h_{1}-h_{4}$ out to $\sim 3 R_{e}$ for a representative sample of 25 nearby ellipticals, using the new stellar kinematics with multiple slits (SKiMS) technique (Norris et al. 2008; Proctor et al. 2008, 2009) with the DEIMOS spectrograph on the $10 \mathrm{~m}$ Keck-II telescope. The data analysis will include triaxial dynamical modeling with the advanced particle-based method NMAGIC (Syer \& Tremaine 1996; de Lorenzi et al. 2006, 2008, 2009). The widefield IFS VIRUS-P has been used to obtain two-dimensional stellar kinematics out to 3-4 $R_{e}$ for several giant ellipticals (Hill et al. 2006; Blanc et al. 2009; Murphy et al. 2009), and multiple pointings of the SAURON spectrograph have been used to measure the stellar LOSVDs out to $\sim 4 R_{e}$ in NGC 3379 and NGC 821 (Weijmans et al. 2009). Surveys using globular clusters (GCs) and planetary nebulae ( $\mathrm{PNe}$ ) as discrete tracers of the distribution function (Romanowsky et al. 2009a; Douglas et al. 2002, 2007; Napolitano et al. 2009; Nantais \& Huchra 2009; Schuberth et al. 2010) can probe much larger radii (out to $\sim 10 R_{e}$ ) and place further constraints on dynamical models (Chaname et al. 2008).

Combined with simulations aimed at establishing the characteristic orbital structure arising from various formation pathways, these observational programs will provide unprecedented insight into the physics of galaxy formation.

\subsection{Orbits in Galactic Potentials}

To deduce the formation histories of galaxies from their orbital structure, we must identify macroscopic groups of orbits that are confined to a well-defined neighborhood of phase space because they followed similar evolutionary pathways. The isolating integrals of motion (or quasi-isolating integrals in the case of perturbed potentials; Contopoulos 1963b; Goodman \& Schwarzschild 1981; Siopis \& Kandrup 2000; Kandrup \& 
Siopis 2003) parameterize the phase space region in which a star remains localized, and encode whatever information about its initial conditions (ICs) is preserved once the system is fully phase-mixed (e.g., Binney \& Tremaine 2008; Binney \& Spergel 1984; Gomez \& Helmi 2010). Orbits which conserve at least one isolating integral per degree of freedom are called regular. It is the ubiquity of regular orbits in galaxies that permits the rich variety in their structure, mirroring their varied formation pathways (Schwarzschild 1979).

The regular orbits in a static potential can be classified into families that conserve qualitatively similar integrals of motion, and therefore have similar morphologies. Which orbital class a given star will occupy is determined by the available phase space for different kinds of orbits in the potential, and its ICs - the phase space need not be uniformly populated. In a time-varying (or otherwise non-ideal) potential such as an ongoing merger, stars diffuse in the space of their conserved integrals, but not completely. If they do not cross boundaries between the orbital families, then their qualitative character may be preserved from the ICs. Crossing between orbital boundaries can serve as a collective relaxation mechanism (e.g., Barnes $\&$ Hernquist 1996). An intuitive grasp of the orbital classes is therefore essential to understanding how dynamical systems evolve and relax.

We begin with a brief overview of the types of regular orbits that are possible in various idealized potentials, leading up to a classification of the orbits in triaxial systems into families that conserve similar integrals. For a more thorough and rigorous presentation we refer the reader to Binney \& Tremaine (2008).

The simplest conceivable model is a spherical potential. In this case, the symmetry about all three Cartesian axes implies conservation of the angular momentum vector, so every orbit is confined to a plane. The star oscillates in radius with frequency $\omega_{r}$ while precessing in azimuth with frequency $\omega_{\theta}$. If these two frequencies are commensurate $\left(m \omega_{r}+n \omega_{\theta}=0\right.$ for some integers $m$ and $n$ ) then the orbit closes on itself, as in a Kepler potential. More generally, the orbits form rosettes that eventually fill an annulus between the minimum and maximum of the radial oscillations (pericenter and apocenter).

Very few galaxies are spherical, but many are consistent with axisymmetry. In an axisymmetric potential the angular momentum component about the symmetry axis, $L_{z}$, is conserved. The direction of $\vec{L}$ precesses about the $z$-axis as $L_{x}$ and $L_{y}$ vary. The radial oscillations no longer return the star to the exact same pericenter and apocenter every cycle, but are still bounded between some $p=r_{\min }$ and $a=r_{\max }$.

Two orbits with the same energy and $L_{z}$ can look quite different from each other, ranging from orbits nearly confined to the $x-y$ plane, resembling eccentric orbits in a thin disk, to puffed-up orbits nearly filling a spherical annulus over long times. This suggests that the orbits are constrained by another integral in addition to $E$ and $L_{z}$, related to how the energy is apportioned into vertical and radial motion. Though it cannot be expressed analytically for a general axisymmetric potential, this third integral $\left(I_{3}\right)$ allows an assortment of stable axisymmetric systems, from thin disks formed by quiescent accretion, to nearly spherical systems heated by persistent perturbations or discrete encounters (e.g., Contopoulos 1960, 1963a; Henon \& Heiles 1964; Saaf 1968; Richstone 1982; Binney \& Tremaine 2008).

However, some galaxies show clear evidence of nonaxisymmetric shapes (e.g., Franx et al. 1991), and simulations of dissipationless violent relaxation in mergers or collapses generically produce triaxial remnants (e.g., van Albada 1982). There is a unique density distribution stratified on similar triaxial ellipsoids whose potential is separable in ellipsoidal coordinates (de Zeeuw \& Lynden-Bell 1985), known as the "perfect ellipsoid." Its distribution function and integrals of motion can be expressed analytically, and its orbital structure has been studied extensively (Stäckel 1890; Eddington 1915; de Zeeuw \& Lynden-Bell 1985; de Zeeuw 1985; Statler 1987). The orbits in the perfect ellipsoid were classified into four major families by de Zeeuw (1985): short-axis tubes (z-tubes) which rotate about the short ( $z$-)axis of the potential, two classes of long-axis tubes ( $x$-tubes) which revolve about the long ( $x$-)axis, and box orbits which behave like perturbed simple harmonic oscillators. Because it is analytic, this model has been widely used as a starting point for understanding the orbital structure of more general triaxial potentials.

Tube orbits resemble the orbits in axisymmetric potentials, and may be thought of loosely as precessing ellipses driven by the bar-like potential of the triaxial ellipsoid (Binney \& Spergel 1982). They conserve angular-momentum-like integrals and therefore avoid the origin of the potential and the zerovelocity surface. It can be shown that $y$-tube orbits rotating about the intermediate axis in the perfect ellipsoid are unstable to vertical perturbations (Heiligman \& Schwarzschild 1979; de Zeeuw 1985), but both $x$-tube and $z$-tube orbits are allowed. Observations of both major- and minor-axis rotation in some elliptical galaxies therefore strongly suggests that these systems are triaxial (Illingworth 1977; Schechter \& Gunn 1979; Binney 1985; Wagner et al. 1988; Franx et al. 1991). $x$-tube orbits are most prevalent in prolate potentials, and are populated by stars with large initial angular momenta about the long axis. $z$-tubes are the dominant type of orbit in oblate systems. Tube orbits oscillate in radii within some bounds $p$ and $a$, and one component of $\vec{L}$ never switches sign. Any net angular momentum of a triaxial system must be carried by the tube orbits, so only these orbits can retain information about a galaxy's initial sense of rotation.

Just as tube orbits may be thought of as precessing ellipses, box orbits may be regarded loosely as axial orbits (or elongated ellipses) librating about the $x$-axis. Binney \& Spergel (1982) illustrated the transition between box and tube orbits using an intuitive toy model. Imagine an elliptical ring lying in a concave, frictionless trough, with its long axis initially parallel to the long axis of the trough, as shown in Figure 1. This configuration allows the ring to lie as low in the trough as possible. Now imagine trying to spin the ring in the trough, by applying an impulsive kick of energy $\frac{1}{2} I \omega^{2}$ to one of its ends. To spin all the way around, the ring must go up and over a barrier, since the curvature of the trough along the perpendicular direction is greater. There is some critical precession frequency $\omega_{c}$ above which the ring will get over the barrier. Below $\omega_{c}$ it will just librate about the equilibrium configuration, with no definite sense of rotation.

The trough's different curvature along the two axes is analogous to triaxiality of a gravitational potential, and the librating mode is analogous to box orbits. If the curvature is the same along both directions perpendicular to the rotation axis (as in an axisymmetric potential), then the energy barrier is zero. Note also that the effective barrier in a triaxial potential is greatest for a star rotating about the $y$-axis, since in this case the difference in curvature along the two perpendicular directions is greatest, making $y$-tubes the most susceptible to instability.

Box orbits are prevalent in triaxial systems with shallow inner density profiles, and conserve integrals similar to the energies 


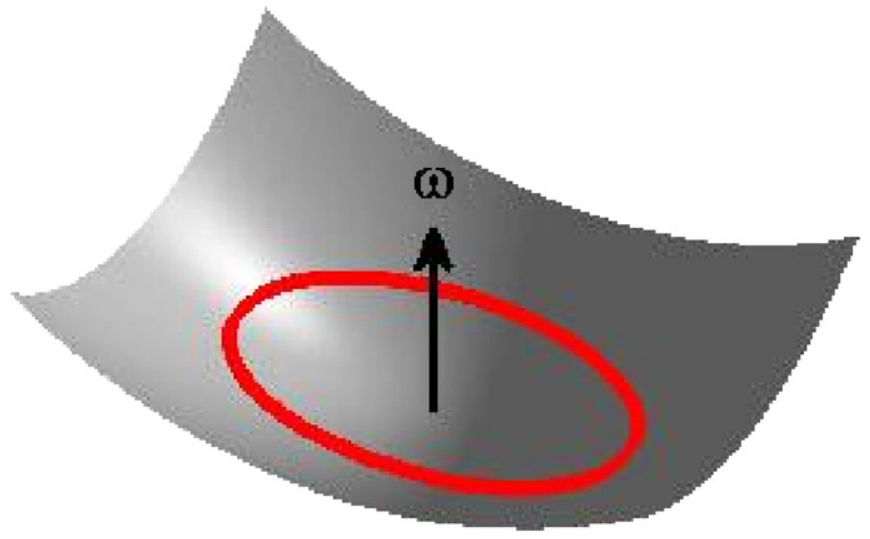

Figure 1. Model of the box-tube boundary: elliptical ring in a frictionless, concave trough. The ring is initially in equilibrium, with its long axis aligned with the long axis of the trough. If an angular velocity $\vec{\omega}$ is imparted to the ring, it must go up and over a barrier to spin all the way around. Above some critical $\omega_{c}$, the ring will make it over the barrier and continue spinning; below $\omega_{c}$ it will just librate about the equilibrium position. This analogue is owed to Binney \& Spergel (1982).

(A color version of this figure is available in the online journal.)

of independent harmonic oscillations about each Cartesian axis. Stars on box orbits have no definite sense of rotation, and can therefore pass arbitrarily close to both the origin (they are "centrophilic") and the zero-velocity surface. Over time, they densely fill a three-dimensional box-like region centered on the origin (de Zeeuw 1985; Statler 1987; Binney \& Tremaine 2008). Powerful nuclear processes such as gas inflow, starbursts, and black hole growth are thought to be major drivers of galaxy evolution, and box orbits may be responsible for conveying information about the rapidly varying central potential to large radii.

Figure 2 shows an example of how the three orbital classes contribute to the projected surface brightness and kinematics in one of our simulated merger remnants. The remnant is shown in projection along the $y$-axis, which maximally separates the orbital classes in two-dimensional space. The boxes and $z$ tubes are elongated along the major axis, while the $x$-tubes are elongated along the minor axis of the projection. All four surface brightness maps are plotted on the same color scale, to show the relative orbital populations at different locations in the sky plane. Note that the isophotes of each individual orbital class appear boxier than the combined isophotes, which are nearly elliptical in shape.

The $x$-tube orbits are responsible for the minor-axis rotation in the remnant, and the $z$-tubes produce the major-axis rotation. There is a striking difference in the amount of streaming of the two classes of tubes: the velocity scale on the $x$-tube map is $\pm 145 \mathrm{~km} \mathrm{~s}^{-1}$, while it is only $\pm 53 \mathrm{~km} \mathrm{~s}^{-1}$ on the $z$-tube map. The remnant therefore appears dominated by minor-axis rotation in the velocity maps, even though $z$-tube orbits dominate its stellar mass.

Two local LOSVDs are shown on the right, at the locations indicated on the maps: one at a point near the center along the major axis (point A) and one near the minor axis farther out (point B). At point A the $z$-tubes have a flat-topped distribution with a high velocity dispersion and negative kurtosis, indicative of canceling streams rotating in opposite directions. The box orbits are strongly peaked at $v=0$, with a large excess in the tails giving the distribution a high positive kurtosis. The combination of the $z$-tube and box orbits yields a combined

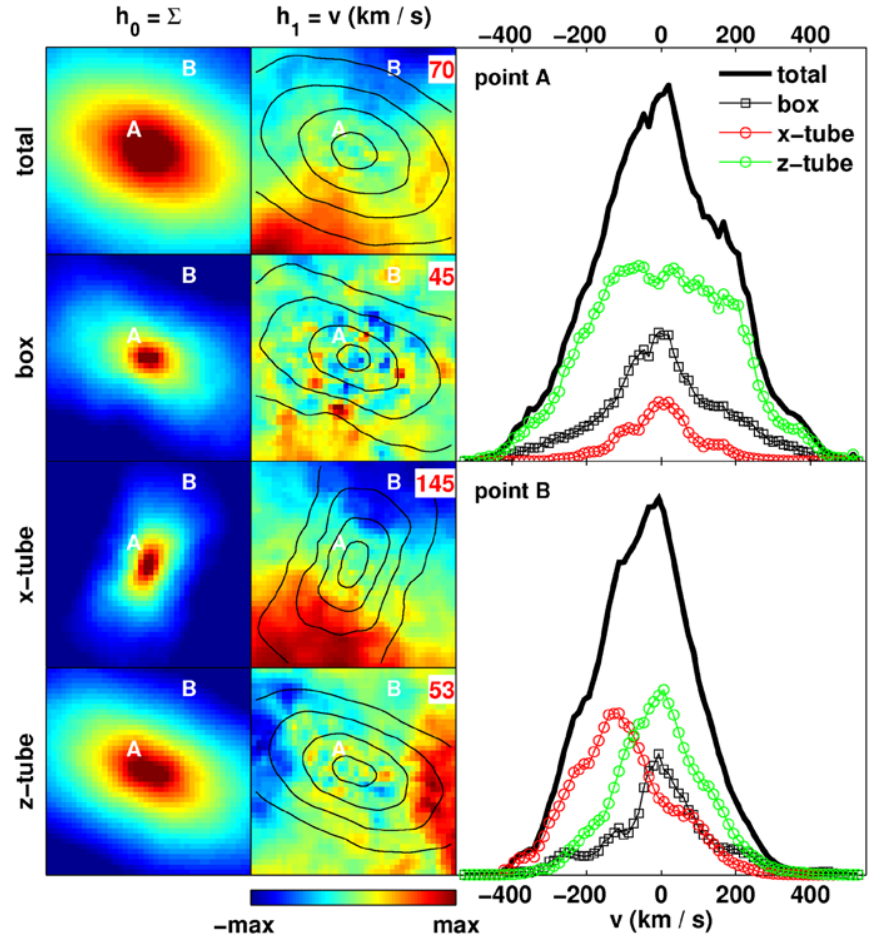

Figure 2. Orbital composition of a typical merger remnant. Remnant $i$ (see Table 1), $15 \%$ gas, viewed along a sight-line near the $y$-axis. First column: projected surface brightness, $\Sigma$, colored on a logarithmic scale. All four maps use the same color scaling to show the relative contribution of each orbital class. Second column: LOS velocity, $v$, with overplotted isophotes. The size of the maps is $1 R_{e} \times 1 R_{e}$, and the color scale runs from $-v_{\max }$ to $v_{\max }$, where $v_{\max }$ is the maximum value of $|v|$ in any pixel. The number in the upper right corner gives the value of $v_{\max }$ in $\mathrm{km} \mathrm{s}^{-1}$. The two right panels show the smoothed LOSVDs at the locations specified by the white letters on the $\Sigma$ and $v$ maps. Top: LOSVD at point A, near the major axis. Bottom: LOSVD at point B, near the minor axis. The heavy black line is the full LOSVD, while the thin lines with markers break it down by orbital class.

(A color version of this figure is available in the online journal.)

LOSVD that is closer to a Gaussian. At point $\mathrm{B}$, the $x$-tube orbits make a larger relative contribution. Their LOSVD is peaked at a high negative velocity and skewed right, indicative of a heated streaming population. The combined distribution has substantial net streaming motion and is slightly skewed in the direction opposite the mean velocity.

An important idealization in the perfect ellipsoid model is that it asymptotes to a flat core. Most elliptical galaxies actually have central density cusps, with slopes ranging from $d \ln \rho / d r \sim 0.5-2.5$ (e.g., Crane et al. 1993; Faber et al. 1997; Lauer et al. 2005, 2007; Kormendy et al. 2009), which may be thought of as perturbations to the perfect ellipsoid potential. These perturbations lead to trapping of box orbits by resonances (e.g., Binney \& Tremaine 2008; Contopoulos \& Mertzanides 1977; Sridhar \& Touma 1996; Collett et al. 1997; Merritt \& Valluri 1999; Tremaine \& Yu 2000), $\vec{n} \cdot \vec{\omega}=0$ for integer $\vec{n}$ (e.g., $z$-tube orbits are trapped by the 1:1 resonance between $\omega_{x}$ and $\left.\omega_{y}\right)$. Stars in an island of phase space around a stable resonance librate around the resonant orbit; these trapped box orbits, called "boxlets," generally avoid the origin (e.g., Carpintero \& Aguilar 1998; Merritt \& Valluri 1999; Fulton \& Barnes 2001). As the perturbation grows larger (the cusp gets steeper), more phase space is occupied by resonant islands until they overlap, producing regions populated by ergodic orbits that eventually fill the entire portion of their five-dimensional energy surface not occupied by regular orbits (e.g., Merritt \& Valluri 1996; Valluri 
\& Merritt 1998; Kandrup \& Siopis 2003; Kalapotharakos et al. 2004).

For simplicity we do not distinguish between regular box orbits, boxlets, and ergodic orbits in this paper, and will loosely use the term "box" to refer to any orbit with no definite sense of rotation. This will be sufficient for the global comparison with observations desired in this study. Spectral analysis of selected orbits (Hoffman 2007; Clozel 2008) reveals that the orbits classified as boxes are generically centrophilic (at least in the inner parts) and stochastic, but they remain fairly localized in phase space over a Hubble time. We defer a detailed study of the nature of the box orbits in the remnants to future work.

The effect of an inner cusp on the orbital populations can be understood by means of a simple experiment-adding a central point mass to a flat-cored, triaxial potential with a large population of box orbits. This "experiment" has been studied extensively, since it has direct astrophysical relevance to black holes in the nuclei of galaxies (e.g., Norman et al. 1985; Gerhard \& Binney 1985; Quinlan et al. 1995; Sigurdsson et al. 1995; Merritt \& Valluri 1997; Merritt \& Quinlan 1998; Valluri \& Merritt 1998; Poon \& Merritt 2001; Holley-Bockelmann et al. 2002; Kalapotharakos et al. 2004). Since box orbits pass arbitrarily close to the origin, they are deflected by the point mass at pericenter passage, causing the orbits to diffuse within box phase space. The velocity changes are primarily along the axis of approach (Chandraskehar 1943), the $x$-axis for box orbits librating about the long axial orbit, so the angular momentum diffusion is mostly in the $y-z$ plane. Eventually the star may wander into $z$-tube phase space (there is no space for $y$-tubes since they are unstable). Since box orbits also strike the zerovelocity surface, they convey information about their pericentric evolution to large radii.

As box orbits diffuse across the $z$-tube boundary, the shape of the potential also becomes more oblate, shrinking the phase space available for boxes and expanding that for $z$-tubes (e.g., Kalapotharakos et al. 2004). A point mass as small as $2 \%-3 \%$ of the total mass can seed this transformation in a flat-cored, triaxial system (Gerhard \& Binney 1985; Merritt \& Valluri 1997; Merritt \& Quinlan 1998; Valluri \& Merritt 1998). When the central mass concentration (CMC) is not an ideal point mass, the diffusion timescale varies with the degree of central concentration-in an $r^{-1}$ cusp, the timescales are typically longer than the lifetime of a galaxy, while in an $r^{-2}$ cusp they are short (Merritt \& Valluri 1996; Merritt \& Fridman 1996; Fridman \& Merritt 1997; Holley-Bockelmann et al. 2001). In hierarchical structure formation, there is an ongoing exchange between processes that induce triaxiality (e.g., violent relaxation in mergers) and gas inflows that deepen the central potential well (e.g., Dubinski 1994). This process therefore undoubtedly plays an important role in galaxy evolution.

\subsection{Gas-rich Mergers}

In current semianalytic models, $\sim 70 \%$ of the stellar mass in present-day ellipticals and classical bulges assembles through major mergers (Hopkins et al. 2009c, 2010; Fakhouri \& Ma 2008; Conroy \& Wechsler 2009). An elliptical galaxy-sized halo has on average undergone $\sim 1$ major merger since $z \sim 2-3$, the epoch during which most of its stellar mass formed (Kauffmann 1996; De Lucia et al. 2006; Hopkins et al. 2008b, 2009c; Stewart et al. 2008). The last major merger was typically between two spiral galaxies, with gas fractions ranging from $\sim 10 \%$ for systems with stellar masses around $3 \times 10^{11} M_{\odot}$, to $\sim 50 \%$ for
$10^{10} M_{\odot}$ systems (Stewart et al. 2009b; Erb et al. 2006 and references therein).

The hypothesis that elliptical galaxies form through mergers between spirals (Toomre \& Toomre 1972) actually preceded the acceptance of the concordance cosmology by about two decades, based on the properties of the galaxies themselves. Gas-rich tidal tails, and rings and shells indicative of the recent disruption of a dynamically cold system, often surround galaxies otherwise resembling ordinary giant ellipticals (Arp 1966; van Dokkum 2005). Early simulations of mergers between disk galaxies could explain a wide variety of the properties of observed ellipticals, including their $r^{1 / 4}$ law density profiles (van Albada 1982; McGlynn 1984; Hernquist 1992; Naab \& Trujillo 2006), slow rotation and anisotropic velocity distributions (Ostriker \& Peebles 1973; Aarseth \& Binney 1978; White 1978; Gerhard 1981), flat rotation curves (White 1978; Farouki \& Shapiro 1982; Efstathiou et al. 1982), fine structure (Hernquist \& Spergel 1992), and apparently triaxial shapes (Gerhard 1981; Barnes 1988; Franx et al. 1991). A simple counting argument based on the numbers of observed interacting pairs and elliptical galaxies in the local universe made the prospect that these pairs turn into ellipticals quite plausible (Toomre 1977).

It was apparent that dissipation must play a large role in mergers long before hydrodynamic simulations with realistic gas fractions became feasible (Negroponte \& White 1983; Hernquist 1989; Nieto et al. 1991; Barnes \& Hernquist 1991; Lutz 1991; Ashman \& Zepf 1992; Hernquist et al. 1993). The measured phase space densities at the centers of elliptical galaxies far exceed the maximum densities in observed spirals, implying a violation of Liouville's theorem unless the initial disks contain $\gtrsim 25 \%-30 \%$ of their mass in gas (Carlberg 1986; Hernquist et al. 1993; Robertson et al. 2006b). Dynamically cold components, such as embedded disks and KDCs, are often observed in elliptical galaxies (e.g., Franx \& Illingworth 1988; Jedrzejewski \& Schechter 1988; Hernquist \& Barnes 1991; Rix \& White 1990; Scorza \& Bender 1995; Jesseit et al. 2007). The high specific frequencies of GCs in ellipticals relative to spirals imply that mergers must trigger the formation of many new clusters from the available gas (Ashman \& Zepf 1992; Fall \& Zhang 2001).

More recent simulations have shown that mergers with $\sim 30 \%$ gas produce remnants that fall on the observed fundamental plane scaling relation (Djorgovski \& Davis 1987; Dressler et al. 1987; Gonzalez-Garcia \& van Albada 2003; Robertson et al. 2006b; Hopkins et al. 2008a), and that the remnants of 1:1 mergers between $40 \%$ gas disks match the one-dimensional kinematic properties of observed ellipticals far better than dissipationless merger remnants (Cox et al. 2006a). Two-dimensional kinematic maps of gas-rich merger remnants display many of the intriguing features seen in real galaxies, including misaligned rotation, central velocity dispersion dips, counter-rotating disks, and KDCs (Jesseit et al. 2007; Barnes 1992; Hernquist \& Barnes 1991). The shapes of the LOSVDs of simulated gas-rich merger remnants display the same trends as ellipticals in the SAURON sample (Bender et al. 1994; Bendo \& Barnes 2000; Naab \& Burkert 2001; Bournaud et al. 2005b; Gonzalez-Garcia et al. 2006; Naab et al. 2006a; Hoffman et al. 2009), and they occupy essentially the same part of the anisotropy-ellipticity $(\delta-\epsilon)$ plane as the SAURON galaxies (with the notable exception of some giant slow rotators that are very round, anisotropic, and featureless; Burkert et al. 2008). These results motivate studies of the intrinsic orbital structure of gas-rich merger remnants, to see to what extent the three-dimensional distribution 
functions of elliptical galaxies can indeed be explained with binary mergers, and shed light on how the observable features arise physically.

To our knowledge, Barnes (1992) was the first to apply orbital analysis in the style of de Zeeuw (1985) and Statler (1987) to simulated merger remnants. He ran a series of dissipationless disk galaxy mergers with varying disk orientations and impact parameters, and classified the stellar orbits in the remnants based on the sign changes in their angular momentum. He found a wide variety in the remnant shapes and orbital structure, depending on the encounter parameters. Some of the remnants displayed substantial orientation twists (see also Gerhard 1983). The mergers often produced both $x$ - and $z$-tube populations with significant net rotation, resulting in large kinematic misalignments. Since the rotation of the majority of observed ellipticals is well aligned with the major axis, he argued that dissipationless disk mergers cannot be the generic way to form elliptical galaxies.

Barnes \& Hernquist (1996) classified the stellar orbits in mergers with $10 \%$ gas, and found that even this small gas component has a dramatic effect on the remnant shapes and orbital structure. Gravitational torques during the merger drain much of the gas of its angular momentum, causing it to collapse inward and form a dense CMC (Hernquist 1989; Barnes \& Hernquist 1991; Mihos \& Hernquist 1994a, 1994b, 1996; Hopkins et al. 2009a), essentially a point mass to stars at $1 R_{e}$. The CMC destabilizes box orbits, leading to a global transformation of the remnant to a more oblate shape. This result is not surprising in light of the studies by, e.g., Gerhard \& Binney (1985) and Merritt \& Quinlan (1998), showing that a central point mass with just $2 \%$ of the total mass can globally transform the structure of a triaxial system.

Jesseit et al. (2005) and Naab et al. (2006a) performed a detailed study of the orbital structure of a large sample of equal and unequal mass merger remnants with $0 \%$ and $10 \%$ gas (Naab \& Burkert 2003), with an emphasis on relating the intrinsic structure to photometric and one-dimensional kinematic observables. They studied the relation between the orbital structure and isophotal shapes in further detail in Jesseit et al. (2008). Using spectral classification (Carpintero \& Aguilar 1998), they found that box orbits typically dominate the inner parts of the dissipationless remnants, while $x$-tubes and $z$-tubes become dominant at larger radii. The box to $z$-tube ratio was the primary determinant of kinematic properties such as the location of the remnants in the $h_{3,4}-v / \sigma$ planes. When a gas component was added, the box population was highly suppressed and the remnants became $z$-tube-dominated and oblate. The shape of the $z$-tube orbits in the more axisymmetric dissipative remnants made the isophotes less boxy. The 1:1 merger remnants were slowly rotating, while the 3:1 remnants were found to be rapidly rotating and disky. They concluded that observed rapidly rotating ellipticals could form from dissipative 3:1 mergers, but boxy, slowly rotating systems (Kormendy \& Bender 1996) could not have formed through dissipative disk mergers.

In this work, we analyze the orbital structure of 1:1 merger remnants in simulations including SF and feedback, enabling us to consider the high gas fractions characteristic of spiral galaxies at $z \sim 2$ (Stewart et al. 2009b; Daddi et al. 2010). At fixed $f_{\text {gas }}$, the effect on the remnant structure may be diminished by including SF and the dissipational features may become more spatially extended, since the gas can be converted to collisionless material early on in the merger and undergo subsequent violent relaxation. We quantify the variation of the orbital structure and intrinsic shape with gas fraction for $f_{\text {gas }}$ ranging from
$0 \%$ to $40 \%$, and discuss the physical mechanisms that may be driving the orbital transformations, with direct comparisons against dynamical models of observed systems in mind. We relate the orbital structure of the remnants to their appearance in two-dimensional kinematic maps on $1 R_{e}$ and $3 R_{e}$ scales, and show that a wide range of different kinematic structures can be accounted for simply by varying $f_{\text {gas }}$ in 1:1 disk mergers.

\subsection{Outline}

In Section 2, we describe our merger simulations and remnant analysis methods, and in Section 3 we present our results. The intrinsic structure of our eight dissipationless remnants is presented in Section 3.1, as a baseline for understanding the effect of adding a gas component. We show how the orbital structure and shape of the remnants varies with $f_{\text {gas }}$ in Section 3.2, and discuss the KDCs and embedded disks that arise in the gas-rich remnants in Section 3.3. In Section 3.4, we briefly describe the structure of the remnants beyond $\sim 1 R_{e}$, deferring a more detailed and quantitative presentation to L. Hoffman et al. (2009a, in preparation). In Section 4, we summarize our results and conclude. The radial profiles of the orbital structure, intrinsic shape, and orientation of all 48 dissipative remnants are presented in the Appendix.

\section{SIMULATIONS AND METHODS}

\subsection{Simulations}

Our galaxy merger simulations were performed using the publicly available TreeSPH (smoothed particle hydrodynamics) code Gadget-2 (Springel 2005; Springel et al. 2001), which uses an advanced formulation of SPH that explicitly conserves both energy and entropy when appropriate (Springel \& Hernquist 2002). In addition to the standard features, our version of the software includes sub-resolution prescriptions for radiative cooling (Katz et al. 1996; Davé et al. 1999), SF, and feedback from supernovae and AGNs, as described in detail in Springel et al. (2005a).

SF and supernova feedback are treated using the multi-phase prescription of Springel \& Hernquist (2003) and Springel et al. (2005a). The interstellar medium (ISM) consists of a cold cloud phase in which stars form, and a hot phase that provides pressure support for the disk. The density dependence of the SF rate is calibrated to match the observed Schmidt-Kennicutt law (Schmidt 1959; Kennicutt 1998). Radiative cooling drives gas transfer from the hot phase to the cold phase, while heating from supernovae feeds gas back into the hot phase. The parameter $q_{\mathrm{EOS}}$ smoothly varies the effective equation of state (EOS) between isothermal $\left(q_{\mathrm{EOS}}=0\right)$ and the pure multi-phase model $\left(q_{\mathrm{EOS}}=1\right)$; higher values of $q_{\mathrm{EOS}}$ correspond to a stiffer EOS and permit stable disks at higher gas fractions. In all of the simulations in this paper we used $q_{\mathrm{EOS}}=0.25$.

The simulations also include sink particles representing black holes, which accrete gas at a rate $\dot{M}_{\mathrm{BH}}=\min \left(\dot{M}_{\text {Bondi }}, \dot{M}_{\text {Edd }}\right)$, where $\dot{M}_{\text {Bondi }} \propto M_{\mathrm{BH}}^{2} \rho_{\text {gas }} / \sqrt{c_{s}^{2}+v^{2}}$ is a rate based on the Bondi-Hoyle-Lyttleton formula (Bondi 1952; Bondi \& Hoyle 1944; Hoyle \& Lyttleton 1939), and $\dot{M}_{\text {Edd }}$ is the Eddington rate. The black holes start with seed masses of $1.4 \times 10^{5} M_{\odot}$, and grow up to masses falling on the observed $M_{\mathrm{BH}}-\sigma$ relation (Gebhardt et al. 2000; Ferrarese \& Merritt 2000; Gultekin et al. 2009) by the end of the merger through gas accretion. A fraction $\epsilon_{f} \epsilon_{r}$ of the accretion energy is released thermally and isotropically into the surrounding gas, where $\epsilon_{r}=0.1$ is the 
assumed radiative efficiency, and $\epsilon_{f}=0.05$ is the fraction of the radiated luminosity that can couple thermally to the gas. Black hole physics has little effect on the global kinematic structure of the remnants, since most of the feedback energy is injected after the merger is complete and most of the stars have already formed (see also Cox et al. 2006a).

The gravitational softening length was $\epsilon=140 \mathrm{pc}$ for the stellar and gas components in our simulations, and $\epsilon=$ $290 \mathrm{pc}$ for the halo component. We decreased the parameter $\eta$ controlling the Gadget-2 time step criterion $(\Delta t=\sqrt{2 \eta \epsilon /|\vec{a}|}$, where $\vec{a}$ is the particle acceleration) from the default value of 0.025 to 0.0025 , to ensure that the steep central cusp that formed in the gas-rich simulations was preserved for several gigayears after the merger.

We set up our merger ICs using standard techniques presented in Springel et al. (2005a), which have been employed in a variety of previous applications (e.g., Di Matteo et al. 2005; Naab et al. 2006a; Hopkins et al. 2006; Robertson et al. 2006b; Cox et al. 2006a; Johansson et al. 2009; Hopkins et al. 2009b). The galaxy models consisted of pure exponential disks embedded in DM halos. For simplicity and ease of comparison with previous work (Cox et al. 2006a; Barnes 1992), stellar bulges were not included in this study. The total mass was specified through the virial velocity, $v_{200}=160 \mathrm{~km} \mathrm{~s}^{-1}$ for an approximately Milky Waysized galaxy, by $M_{\mathrm{tot}}=v_{200}^{3} /\left(10 \mathrm{GH}_{0}\right)$. The halo was modeled as a Hernquist (1990) profile with spin parameter $\lambda=0.033$, and scale radius chosen to match an Navarro-Frenk-White profile with concentration $c=0.9$ in the inner parts.

The disk mass was set to $M_{d}=f_{d} M_{\text {tot }}$, with $f_{d}=0.041$. Its scale length of $r_{d}=3.9 \mathrm{kpc}$ was determined by the requirement that it be centrifugally supported with the same specific angular momentum as the halo, $J_{d}=f_{d} J_{\text {tot }}$. The stellar component of the disk was assigned a radially constant vertical scale height of $0.2 r_{d}$, while the vertical structure of the gas component was set by the requirement of hydrostatic equilibrium. The disk models were realized with 80,000 equal-mass particles, a fraction $1-f_{\text {gas }}$ of them in collisionless stars and the other $f_{\text {gas }}$ in SPH particles. The halo was comprised of 120,000 collisionless DM particles.

For each simulation two identical disk galaxies were constructed following this procedure, and placed on a parabolic orbit with an impact parameter of $7.1 \mathrm{kpc}$. At their initial separation of $140 \mathrm{kpc}$, the center-of-mass trajectories were well approximated by point mass orbits. At least in the inner parts, the final structure is not highly sensitive to our choice of small impact parameter, since the most bound particles (the stars) lose most of their orbital angular momentum to the halo by the time the cores merge even in wide encounters (Barnes 1998; Cox et al. 2006a). The effect of the merger impact parameter on the stellar halo structure will be explored in future work (L. Hoffman et al. 2009a, in preparation).

The orientation of the disks relative to the merger $(x-y)$ plane was parameterized by the two angles $\theta$ and $\phi$, the polar angle of the spin vector relative to the $z$-axis and its azimuthal angle relative to the axis of approach, as shown in Figure 6 of Toomre \& Toomre (1972). We used the scheme proposed by Barnes (1992) to sample the space of possible disk orientations in a relatively unbiased way, including prograde, retrograde, and polar orbits. The spin of the first disk coincides with each of the four symmetry axes of a regular tetrahedron pointing upward, while that of the second disk points along the axes of the corresponding downward-pointing tetrahedron. The resulting eight merger orbits are listed in Table 1. This set of encounter
Table 1

Merger Orbits

\begin{tabular}{lrrrr}
\hline \hline Orbit & \multicolumn{1}{c}{$\theta_{1}$} & $\phi_{1}$ & $\theta_{2}$ & \multicolumn{1}{c}{$\phi_{2}$} \\
\hline$i$ & 0 & 0 & 71 & 30 \\
$j$ & -109 & 90 & 71 & 90 \\
$k$ & -109 & -30 & 71 & -30 \\
$l$ & -109 & 30 & 180 & 0 \\
$m$ & 0 & 0 & 71 & 90 \\
$n$ & -109 & -30 & 71 & 30 \\
$o$ & -109 & 30 & 71 & -30 \\
$p$ & -109 & 90 & 180 & 0 \\
\hline
\end{tabular}

orbits has been used in a number of previous studies (e.g., Barnes 1992; Naab \& Burkert 2003; Naab et al. 2006a; Cox et al. 2006a), which may be used as a reference point for comparison with our results where appropriate. The eight encounters listed in Table 1 were repeated at seven different gas fractions, $f_{\text {gas }}=0 \%, 5 \%, 10 \%, 15 \%, 20 \%, 30 \%$, and $40 \%$, for a total of 56 merger simulations.

Once on their orbits, the galaxies reach their first pericenter passage at $t \approx 0.35 \mathrm{Gyr}$, at which point their morphologies become strongly distorted by tidal forces from the other galaxy, and the stars and gas each form a bar. The stellar bar slightly trails the gas bar, torquing back on the gas and draining its angular momentum so that the gas flows inward and undergoes a burst of star SF (Mihos \& Hernquist 1994a; Barnes \& Hernquist 1996; Escala et al. 2004; Hopkins et al. 2009b). At $t \approx 1.8 \mathrm{Gyr}$, the galaxies reach second passage, and their cores merge shortly thereafter. The strong and persistent gravitational torques during the second passage and final merger typically induce a stronger central starburst than at first passage. By about $0.5 \mathrm{Gyr}$ after the merger the size, shape, and velocity dispersion of the remnant (measured within $\sim 1 R_{e}$ ) reach a steady state (Cox et al. 2006a), and the system may be considered relaxed.

\subsection{Remnant Analysis}

We freeze the potential at $t=4.3 \mathrm{Gyr}$ (about $2.5 \mathrm{Gyr}$ after the merger is complete), and represent it using the bi-orthogonal "designer" basis expansion (Clutton-Brock 1972, 1973; Binney \& Tremaine 2008) presented in Hernquist \& Ostriker (1992),

$$
\begin{aligned}
\rho(r, \theta, \phi) & =\sum_{\mathrm{nlm}} A_{\mathrm{nlm}} \rho_{n l}(r) Y_{l m}(\theta, \phi) \\
\Phi(r, \theta, \phi) & =\sum_{\mathrm{nlm}} B_{\mathrm{nlm}} \Phi_{n l}(r) Y_{l m}(\theta, \phi) .
\end{aligned}
$$

The terms in this expansion individually satisfy Poisson's equation, and the lowest-order term is a Hernquist (1990) profile, which should be a good first approximation to the density profile of a remnant resembling an elliptical galaxy. Before computing the expansion coefficients we symmetrize the particle distribution by reflecting all of the positions and velocities about the origin, effectively increasing the particle statistics by a factor of two and reducing global fluctuations. The orbit of each stellar particle is then integrated through $\sim 300$ radial turning points (peri/apocenter passages) in the static potential using a Bulirsch-Stoer integrator (Bulirsch \& Stoer 1966; Press et al. 1992). This technique allows all of the stellar orbits to be efficiently followed for $\sim 150$ dynamical times, including halo orbits for which this corresponds to many Hubble times. It also lessens spurious relaxation and simplifies the orbital analysis by fixing the principle axes of the system. 
Each stellar orbit was classified as a box, $x$-tube, or $z$-tube orbit using a simple algorithm based on the sign changes in the star's angular momentum, introduced by Barnes (1992). At each time step we computed the angular momentum vector $\vec{j}$, and checked whether each component of $\vec{j}$ had changed sign since the last step. At the end of the integration we constructed the vector $\vec{k}$ such that $k_{i}=1$ if $j_{i}$ never changed sign, and $k_{i}=0$ otherwise. The orbit was assigned the classification code $C=k_{x}+2 k_{y}+4 k_{z}$, which is 0 for a box orbit, 1 for an $x$-tube orbit, and 4 for a $z$-tube orbit. For a perfect triaxial ellipsoid, other values of $C$ are unphysical.

This classification scheme depends on a correct specification of the system orientation, and many of the remnants have intrinsic orientation twists. We therefore diagonalized the inertia tensor, in the form $I_{i j}=\sum x_{i} x_{j}$ (e.g., Aguilar \& Merritt 1990), in $\sim 50$ cumulative energy bins, and used the local orientation based on the star's energy for the classification. The eigenvectors of $I_{i j}$ are the principal axes of the remnant's figure, and the square roots of the eigenvalues give the relative scale lengths along the three principal axes, $a, b$, and $c$. All particles-gas, stars, and DM-were included in $I_{i j}$, since a star's orbit is determined by the sum of all three components. Note that since we used the local orientation for the classification, e.g., the position angle of the $z$-tube rotation may appear to vary with galactocentric radius in the remnants with significant orientation twists.

Only $\sim 1 \%$ of the stellar orbits were not assigned $C$ values of 0,1 , or 4 , except in rare instances of sudden orientation twists or locations where the potential was very nearly spherical. The results of our simple classification scheme were consistent with spectral classification (Carpintero \& Aguilar 1998; Binney \& Spergel 1982, 1984; Hoffman 2007), and we determined that the simpler algorithm was better suited for the noisy potentials and gross analysis desired in this work. However, note that this algorithm does not distinguish between boxes, resonant boxlets, and ergodic orbits.

For each remnant, we present radial profiles of the orbital structure and intrinsic shape as shown for one example in Figure 3. First we order the stellar particles by energy, and divide them into $\sim 20$ energy bins with equal numbers of particles. The mass fraction of stars in box, $x$-tube, and $z$-tube orbits is computed for each energy bin, and plotted versus percentile in binding energy (by mass), as in the left-hand panel of the figure. For ease of comparison with observational results, we also compute the mean galactocentric radius of the particles in each energy bin, and label the bins corresponding to $0.3,1,3$, and the largest integer number of $R_{e}$.

To understand kinematic maps, it is useful to quantify not only the populations of the major orbital classes, but also their degree of streaming. For this purpose, we plot profiles of

$$
\beta=\left(N_{+}-N_{-}\right) /\left(N_{+}+N_{-}\right)
$$

for the $x$-tube and $z$-tube orbits, as shown in the left center panel. Here $N_{+}$and $N_{-}$are the numbers of particles rotating in either direction; $\beta=0$ corresponds to perfect cancellation of the rotation, while $\beta= \pm 1$ means that all of the stars are streaming in the same direction. The sign convention was chosen by requiring $\beta$ to be positive at the 40th percentile in binding energy $\left(1 R_{e}\right)$.

The intrinsic shape and orientation vector are computed by diagonalizing the inertia tensor as described previously. We plot the maximum and minimum axis ratios, $\epsilon=c / a$ and $r_{\min }=\min (b / a, c / b)$, and the triaxiality parameter,

$$
T=\left(a^{2}-b^{2}\right) /\left(a^{2}-c^{2}\right),
$$

in the right center panel of Figure 3. $T$ ranges from 0 for a perfectly oblate spheroid to 1 for a prolate spheroid. In the right-hand panel, we plot the polar and azimuthal angles $\left(\theta_{\text {symm }}\right.$ and $\left.\phi_{\text {symm }}\right)$ of the symmetry axis-the long axis if $T>0.5$ within $1 R_{e}$, or the short axis if $T<0.5$. If the short-axis orientation is plotted then we color the lines red, otherwise we color them blue. The shape of a remnant that is oblate within $1 R_{e}$ may become prolate farther out, in this case, we still plot the orientation of the short axis at all radii, which does not make the angles ill-defined since the remnants are never too close to axisymmetric at large radii. Note that $\theta_{\text {symm }}$ and $\phi_{\text {symm }}$ represent angles between a vector and an axis (whose direction is defined only up to a sign), and therefore range from 0 to $90^{\circ}$. When plotting the orientation profiles, we use a fixed coordinate system where the $z$-axis points along the disks' initial orbital angular momentum vector, and the $x$-axis is along the initial direction of approach.

On the same axes we plot the intrinsic kinematic misalignment,

$$
\Psi_{\text {kin }}=\arctan \left|j_{z} / j_{x}\right|,
$$

or the angle between the short axis and the net angular momentum vector. To compute $\Psi_{\text {kin }}$ we revert back to the local principal axes system, so that $\Psi_{\text {kin }}=0$ always corresponds to rotation about the intrinsic short axis.

To place our work in an observational context, it is also useful to show how the intrinsic orbital structure imprints itself on SAURON-like two-dimensional kinematic maps. We constructed histograms of the line-of-sight (LOS) velocity in $40 \times 40$ spatial bins within $1 R_{e}$, using 80 velocity bins within $\pm 3.5 \sigma$. For each LOSVD, we performed a least-squares fit to the five-parameter function

$$
F(y)=A e^{-w^{2} / 2}\left[1+h_{3} H_{3}(w)+h_{4} H_{4}(w)\right],
$$

where $w=(y-v) / \sigma$, and $v$ and $\sigma$ represent the mean velocity and velocity dispersion. Here $\mathrm{H}_{3}$ and $\mathrm{H}_{4}$ are Gauss-Hermite (GH) polynomials, and the parameters $h_{3}$ and $h_{4}$ measure the skewness and kurtosis of the distribution (van der Marel \& Franx 1993; Gerhard 1993; Binney \& Merrifield 1998). The Gadget particles were smoothed over a radius $h_{\text {smooth }}=\max \left(h_{\text {see }}, h_{\text {ngb }}\right)$, where $h_{\text {see }}=150 \mathrm{pc}$ corresponds to a seeing of 1.5 " at $20 \mathrm{Mpc}$, and $h_{\mathrm{ngb}}$ is 1.7 times the distance to the 128th nearest neighbor.

We focus primarily on the velocity fields in this work. Note that $v$, as derived from the fit of Equation (4), deviates systematically from the true mean of the distribution for nonzero $h_{3}$ and $h_{4}$ (see van der Marel \& Franx 1993 for the relevant correction terms). However, we follow the convention of previous authors (e.g., Bender et al. 1994) and simply use $v$ from the GH fit to represent the velocity in this paper.

When displaying all of the remnants at a given $f_{\text {gas }}$, we choose the viewing angles randomly (from an isotropic distribution) to give a fair representation of their appearance in kinematic maps. We label each map with the LOS angles $\theta$ and $\phi$, where $\theta$ is the polar angle between the LOS and the $z$-axis, and $\phi$ is the azimuthal angle between the LOS and the $y$-axis. The apparent major-axis rotation is maximized when $\theta=90^{\circ}$, and the minoraxis rotation is maximal at $\phi=0$ for a given $\theta$. 


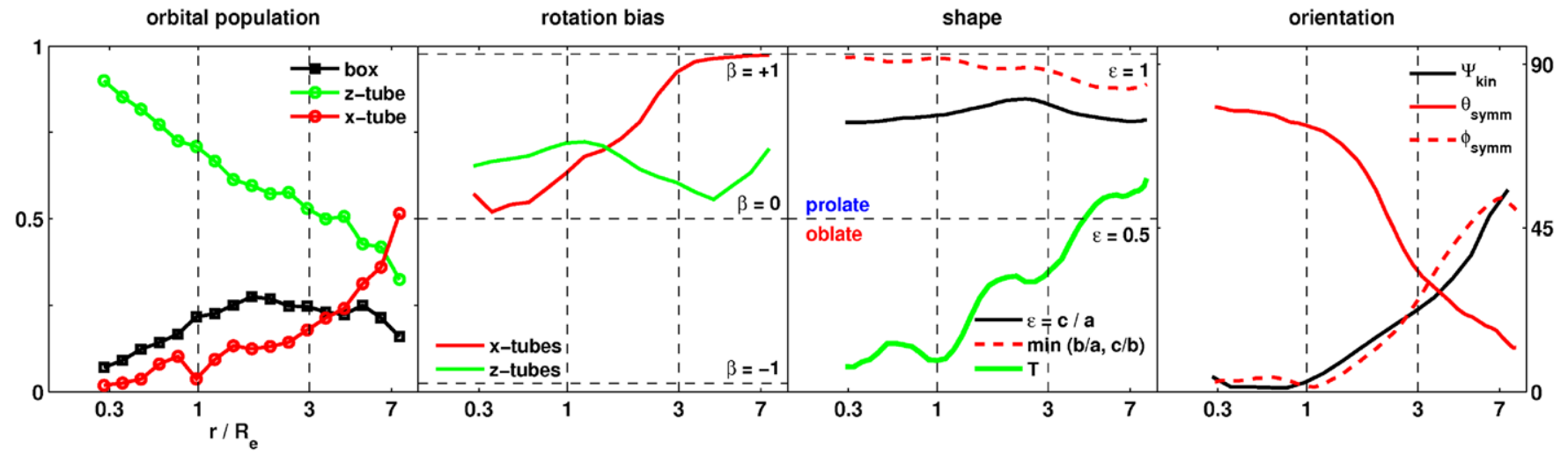

Figure 3. Radial profiles of the orbital structure and intrinsic shape of remnant $i, 40 \%$ gas. Left: fraction of the stellar mass in each of the three major orbital classes vs. percentile in binding energy. The horizontal axis is labeled by the mean radius (in units of $R_{e}$ ) of the stars in the current energy bin (see the text for details). Left center: rotation bias, $\beta=\left(N_{+}-N_{-}\right) /\left(N_{+}+N_{-}\right)$, of the $x$-tube and $z$-tube orbits. $\beta=0$ corresponds to perfect cancellation of the rotation, while $\beta= \pm 1$ means that all of the stars are streaming in the same direction. We choose the overall sign so that $\beta$ is positive at the 40th percentile in binding energy ( $\left.1 R_{e}\right)$. Right center: intrinsic shapes of the remnants. The heavy green line is the triaxiality parameter, $T$, for all particles in the current energy bin and more bound. The black line is the intrinsic ellipticity $\epsilon=c / a$, and the red dashed line is the smaller of the other two axis ratios. The horizontal line at $T=0.5$ marks the boundary between oblate and prolate shapes. Both stars and DM particles are included in the evaluation of the intrinsic shapes. Right: intrinsic orientations $\left(\theta_{\text {symm }}, \phi_{\text {symm }}\right)$ and kinematic misalignments $\left(\Psi_{\mathrm{kin}}\right)$, in degrees. The red solid and dashed lines are the polar $\left(\theta_{\text {symm }}\right)$ and azimuthal $\left(\phi_{\text {symm }}\right)$ angles of the symmetry axis, in this case the short axis since the remnant is oblate. In general, we will plot $\theta, \phi$ of the short axis in red if the remnant is oblate $(T<0.5)$ at $1 R_{e}$, and $\theta, \phi$ of the long axis in blue if the remnant is prolate $(T>0.5)$ at $1 R_{e} . \theta$ and $\phi$ are evaluated in a coordinate system where the disks' initial orbital angular momentum points in the $\hat{z}$-direction, and the galaxies initially approach one another along the $x$-axis. To evaluate $\Psi_{\text {kin }}$ (Equation (3); black line), we return to the local principal axes coordinate system. In all panels, the $1 R_{e}$ scale probed by SAURON and the $3 R_{e}$ scale typical of SKiMS data are marked with vertical lines.

(A color version of this figure is available in the online journal.)

\section{RESULTS AND DISCUSSION}

\subsection{The Dissipationless Remnants}

The structure of the dissipationless remnants is presented in Figure 4. There is substantial variation in the orbital distribution and shape over the eight merger orbits-for instance remnant $j$ is uniformly prolate and dominated by $x$-tube orbits, while remnant $o$ is oblate-triaxial and composed primarily of box orbits and $z$-tubes. Overall, the remnants are dominated by box orbits in their inner parts $\left(r \lesssim 1.5 R_{e}\right)$, and tube orbits in their outskirts. This is not surprising since the Hernquist (1990) profile, which generically provides a good fit to the density profiles of dissipationless merger remnants, has a shallow inner cusp $\left(\rho \propto r^{-1}\right)$ and much steeper outer profile $\left(\rho \propto r^{-4}\right)^{6}$.

The inner shapes of the remnants are typically prolate-triaxial and highly flattened $(T \sim 0.8, \epsilon \sim 0.6)$, consistent with previous results for head-on mergers of stellar systems that have shed most of their orbital angular momentum to the halo (Miller \& Smith 1980; White 1983; Barnes 1992; Cox et al. 2006a). Farther out the remnants become rounder $(\epsilon \sim 0.8)$ and closer to maximally triaxial. See also Dubinski (1994); Kazantzidis et al. (2004); Debattista et al. (2008); Valluri et al. (2010); Abadi et al. (2010); and Romano-Diaz et al. (2009), who studied halo shapes in a cosmological context and found that the response of the DM to baryonic condensation into galaxies drives the halos to rounder and more oblate shapes.

There is substantial cancellation of the prograde and retrograde rotation of the $z$-tube orbits ( $\beta$ near zero), especially in the inner parts. On the other hand, the $x$-tube populations are highly streaming, with $\beta$ typically approaching one at large radii. Remnant $j$ is a notable exception to this rule, with the rotation of its dominant $x$-tube orbits canceling nearly perfectly over

\footnotetext{
6 A star in what we are calling the "outer parts" $\left(\sim 3-6 R_{e}\right)$ still occupies the inner DM halo (which also follows roughly a Hernquist 1990 profile but with a much larger scale radius). The total density (stars $+D M$ ) at these radii scales roughly like $r^{-2}$-still steep compared to $r^{-1}$, but not nearly as steep as $r^{-4}$.
}

the full range of radii plotted. Upon closer examination of this remnant, it turns out that this owes to the symmetry of the ICs. Both disks on orbit $j$ are substantially inclined, but their spins point in opposite directions. If the $x$-tube orbits in the remnant are separated by their disk of origin, nearly all of those from disk 1 rotate in one sense about the long axis, while those from disk 2 rotate in the opposite sense, giving the net cancellation seen in Figure 4.

The dissipationless remnants do not show strong orientation twists, and their long axis (which is typically the axis of symmetry, since the remnants are prolate) lies in the merger plane $\left(\theta_{\text {symm }}=90^{\circ}\right)$, along the axis of the final coalescence of the stellar components. This is consistent with the interpretation of Novak et al. (2006): the time-varying tidal field along the merger axis couples coherently to the stellar orbits, producing a bounce that elongates the remnant along the direction of tidal compression (Miller 1978; White 1983). The kinematic misalignments are large since the net rotation tends to favor the long axis, and noisy since the magnitude of the rotation is often small.

In the $1 R_{e}$ kinematic maps, the dissipationless remnants are generally slowly rotating (e.g., remnants $j$ and $p$ ) or are dominated by minor-axis rotation (e.g., remnants $m$ and $n$ ). Some of the remnants (e.g., $p$ and $l$ ) definitely show both major and minor axis rotation, though small in magnitude $(v / \sigma \sim 0.15)$.

Figure 4 is a baseline for understanding how a gas component affects the remnants-it may be compared with Figures 15-20 to see how the orbital structure evolves for each merger orbit as $f_{\text {gas }}$ is increased from $0 \%$ to $40 \%$.

\subsection{Variation with Gas Fraction}

In Figure 5, we illustrate the effect of dissipation on the remnant structure by varying the gas fraction for a fixed merger orbit, $i$, whose structural transformations are representative of the overall trends. Case $i$ is an encounter between a prograde disk in the merger plane $\left(\theta_{1}=\phi_{1}=0\right)$, and a highly inclined 


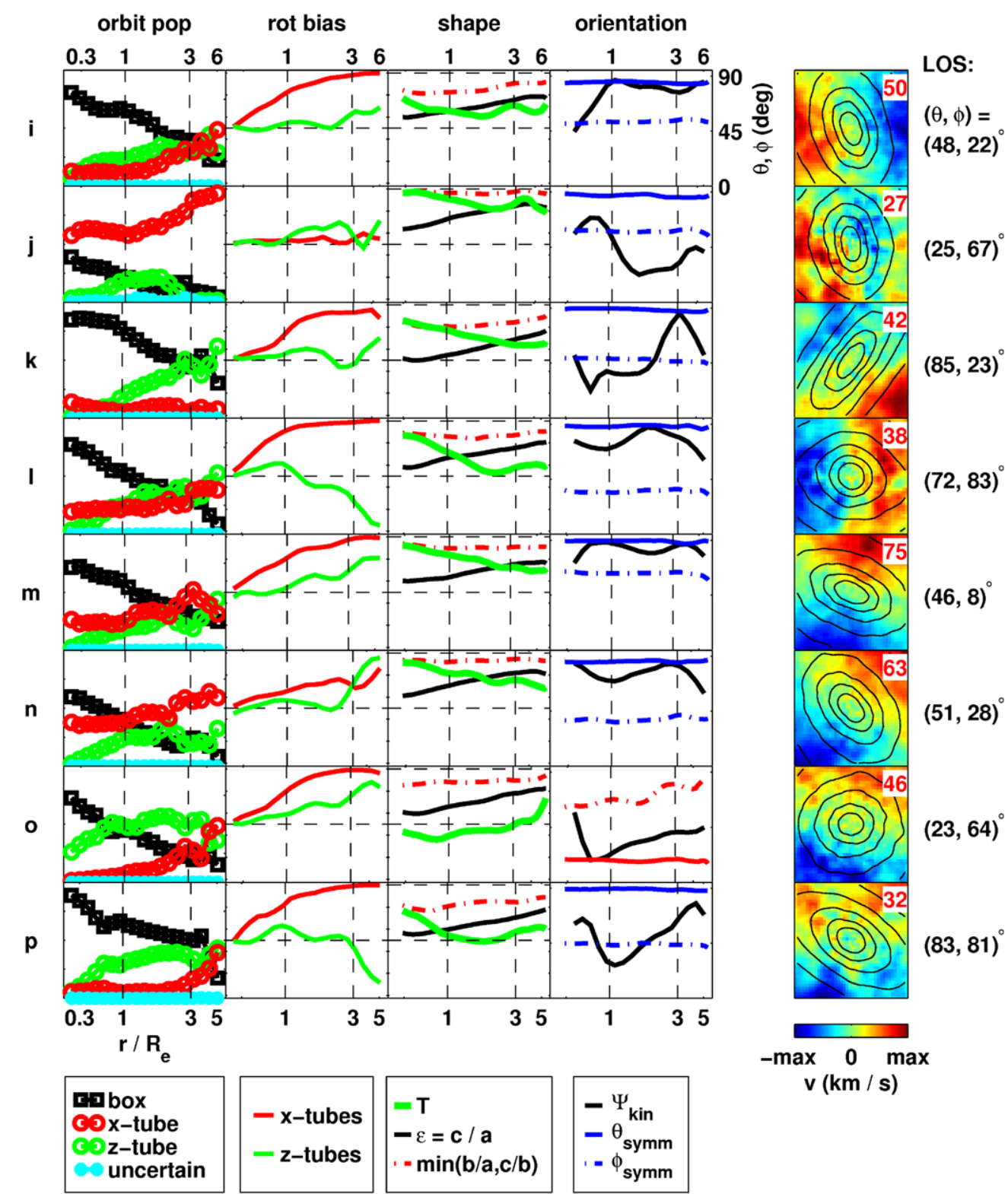

Figure 4. Intrinsic structure of the dissipationless remnants. The first four columns follow the format of Figure 3 . The merger orbit (see Table 1) is specified on the left for each row. In the right-hand column we show a $1 R_{e}$ velocity map of the remnant, viewed along a randomly (isotropically) chosen LOS. The two angles specifying the LOS are indicated by the labels on the right. $\theta$ is the polar angle between the LOS and the short axis (this axis was chosen as the reference throughout the paper since most of the remnants with gas are oblate), and $\phi$ is the azimuthal angle between the LOS and the intermediate axis. The major-axis rotation is maximal at $\theta=90^{\circ}$, and the minor-axis rotation is maximized at $\phi=0$ for fixed $\theta$. The velocity scale runs from $-v_{\max }$ to $v_{\max }$, where $v_{\max }$ is the maximum value of $|v|$. The value of $v_{\max }\left(\right.$ in $\mathrm{km} \mathrm{s}^{-1}$ ) is indicated by the label in the upper right corner of each map.

(A color version of this figure is available in the online journal.)

disk that is also slightly prograde $\left(\theta_{2}=71^{\circ}, \phi_{2}=30^{\circ}\right)$. The orbital structure, shape, and orientation profile of this remnant are shown for seven different gas fractions in Figure 5. Unlike in Figure 4, the velocity maps are all in projection along the $y$-axis, to clearly show the rotation of both the $x$-tube and $z$-tube orbits and how it varies with $f_{\text {gas }}$.

As $f_{\text {gas }}$ increases from $0 \%$ to $40 \%$, the population of box orbits within $\sim 1.5 R_{e}$ declines, and the $z$-tube population increases. The most rapid change in the orbital populations occurs between $0 \%$ and $20 \%$ gas. The outer orbital structure $\left(r \gtrsim 2 R_{e}\right)$ is relatively unaffected by the gas, and the $x$-tube population does not display an obvious trend.

The variation in the intrinsic shape closely follows the change in the orbital populations. As $f_{\text {gas }}$ increases, the inner parts become progressively more oblate; the $30 \%-40 \%$ gas remnants are nearly oblate axisymmetric within $1 R_{e}(T \lesssim 0.1)$. The remnants also become rounder with higher $f_{\text {gas }}: \epsilon$ increases from $\sim 0.6$ for the dissipationless remnant to $\sim 0.8$ at the highest gas fractions.

Though the mass in $z$-tube orbits within $\sim 2 R_{e}$ rises substantially as $f_{\text {gas }}$ increases from $0 \%$ to $15 \%$, the remnants show little change in their major-axis rotation over this range, since the mass in prograde and retrograde orbits is nearly equal $(\beta \sim 0)$. The $15 \%$ gas remnant, though dominated by $z$-tubes within $1 R_{e}$, still appears as a minor-axis rotator in the projected velocity map.

In the $20 \%$ gas remnant, the innermost $z$-tube orbits begin to show substantial streaming, in a sense opposite the net rotation 


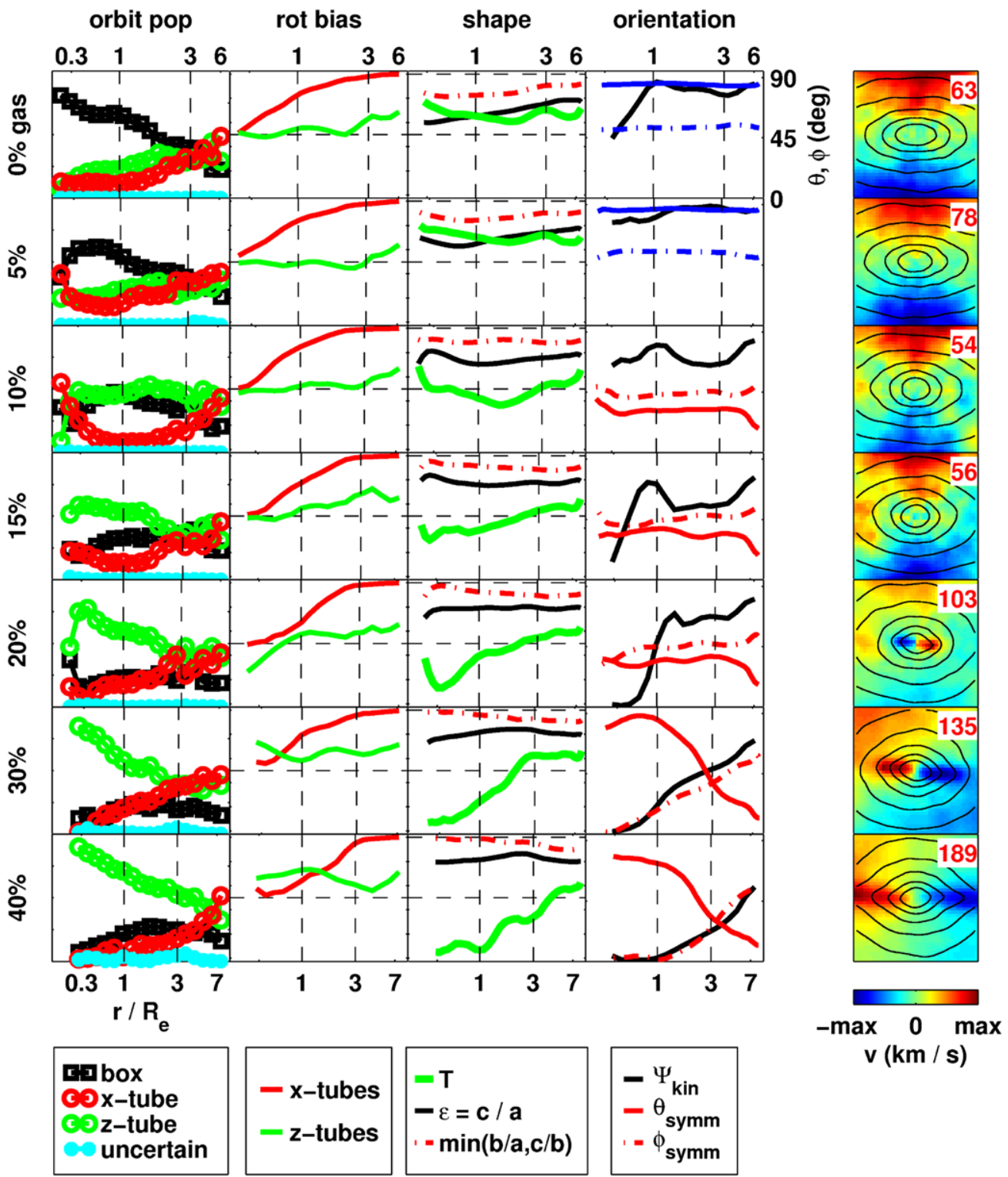

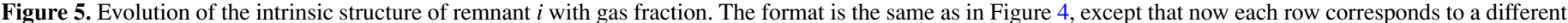

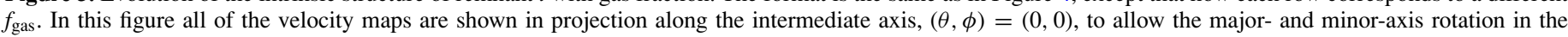
remnants at different $f_{\text {gas }}$ to be compared directly.

(A color version of this figure is available in the online journal.)

farther out. This inner streaming shows up as a prominent KDC in the velocity map. Note that the minor-axis rotation present at lower gas fractions is still there in this map, but it is dwarfed on the velocity scale by the rapid rotation within the KDC. In the 30\%-40\% gas remnants, the $z$-tube orbits show significant streaming $(\beta \sim 0.3)$ over the full range of radii plotted, and the edge-on kinematic maps display rapid, disk-like major-axis rotation. This rapid rotation about the intrinsic short axis gives kinematic misalignments near zero in the inner parts.

Although the rotation bias of the $z$-tube orbits in the $30 \%$ gas remnant appears fairly uniform, the cause of the streaming in the inner and outer parts is quite different. In the outer parts the net angular momentum is retained from the ICs, and is therefore present at all gas fractions. The inner streaming is a direct consequence of dissipation - the gas that retains its angular momentum during the merger re-forms a cold disk as it dissipates energy, which forms new stars primarily after the violent relaxation of the merger is complete. Thus the inner part of the gas-rich remnants is a superposition of a hot, slowly rotating component consisting of the stars present before the merger (hereafter referred to as "old stars"), and a maximally rotating disk composed of "new stars" formed through dissipation. This is why $\beta$ is only around 0.3 despite the high maximum velocities in the maps. We will elaborate on this point in Section 3.4.

The inner parts of the gas-rich remnants are often rapidly tumbling, which produces large intrinsic orientation twists between $\sim 1$ and $3 R_{e}$, as in the bottom two rows of in Figure 5. Note that the orientations in the inner parts of these remnants are an arbitrary snapshot in time; had the simulation been frozen earlier or later $\theta_{\text {symm }}$ and $\phi_{\text {symm }}$ could have quite different values.

In the preceding discussion we have demonstrated that the low- $f_{\text {gas }}$ remnants show little major-axis rotation not because they lack $z$-tube orbits, but because there is a large degree of 


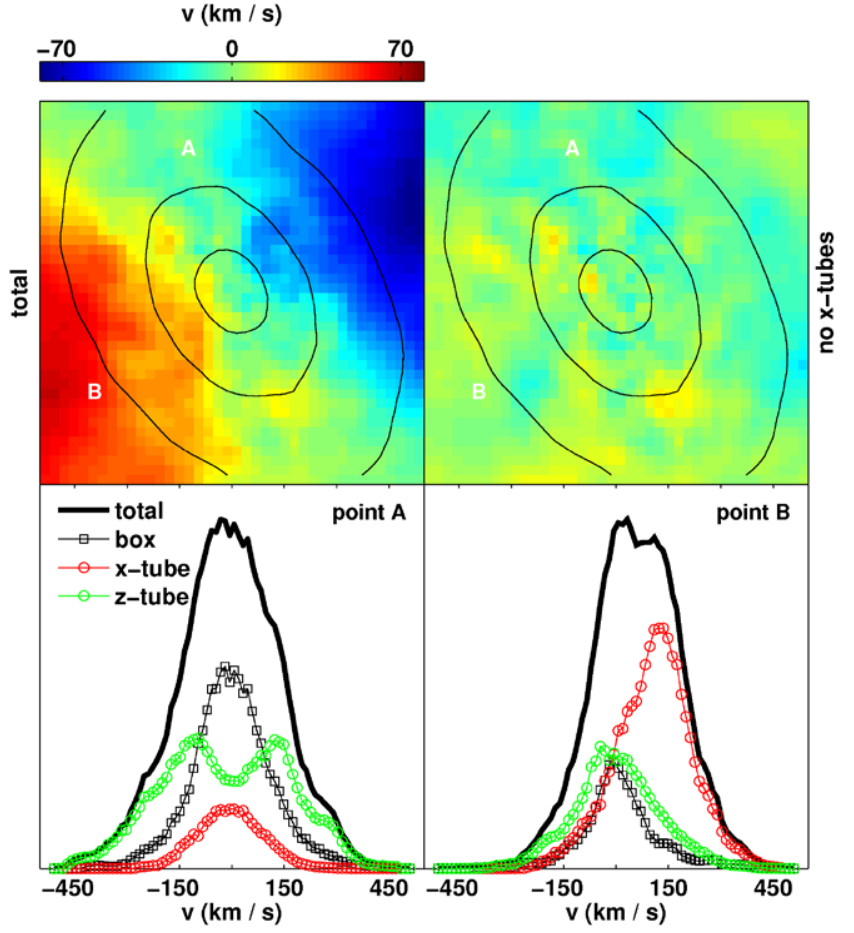

Figure 6. Cancellation of the $z$-tubes and streaming of the $x$-tube orbits. Upper left: velocity map of remnant $m, 10 \%$ gas, viewed in a near-intermediate axis projection. Upper right: same map with $x$-tube orbits removed. Lower left: LOSVD at location A, near the major axis. Lower right: LOSVD at location B, near the minor axis. The velocity scale is the same on both maps.

(A color version of this figure is available in the online journal.)

cancellation between the $z$-tubes rotating in opposite directions. An observer who measured the major- and minor-axis rotation speeds of the typical $10 \%$ gas remnant might naively think that the system was prolate in shape and dominated by $x$-tube orbits, when in fact the predominance of the minor-axis rotation is caused by a sub-dominant population of $x$-tubes that are highly streaming.

We highlight this point in Figure 6 showing remnant $m$ at $10 \%$ gas, which has comparable masses in $x$-tubes and $z$-tubes within $1 R_{e}$, but somewhat more $z$-tubes (see Figure 16). The upper left panel is a velocity map of the remnant in a nearly edge-on projection (maximizing both the major- and minor-axis rotation), and the second panel shows the same map with the $x$-tube orbits removed. Without the $x$-tubes, the remnant shows almost no rotation at all. The LOSVDs at the locations marked by the white letters are plotted in the lower two panels. The lower left panel clearly shows the canceling streams of $z$-tube orbits along the major axis, while the lower right panel depicts the highly streaming $x$-tubes along the minor axis, peaked at nearly $200 \mathrm{~km} \mathrm{~s}^{-1}$. In the lower left panel, the large population of box orbits fills in the gap in the bimodal $z$-tube distribution, yielding a total LOSVD that is nearly Gaussian in shape.

Figure 7 provides insight into the physical explanation for this orbital structure by mapping out the locations, in the original disks, of the stars that end up in each of the three orbital classes in remnant $i$, with $f_{\text {gas }}=0 \%, 15 \%$, and $30 \%$. Disk 1 is the direct disk, and disk 2 is the inclined one. Only old stars are included in this plot, so the dissipative disk component in the $30 \%$ gas remnant is left out. Each disk was partitioned into cylindrical bins containing 300 stars apiece, and the bins were labeled according to which orbital class formed the plurality of stars among those 300 in the final remnant-black for boxes, green for $z$-tubes, and red for $x$-tubes. The bins favoring tube orbits were further labeled based on whether they tended to stream in one direction in the final remnant, or to have canceling rotation. Bins with $\beta>0.3$ are indicated with upward-pointing arrows and labeled as "counterclockwise," while those with $\beta<-0.3$ are indicated with downward-pointing arrows and labeled as "clockwise." Bins with $|\beta|<0.3$ are labeled as "canceling" and indicated with filled circles. The blue circles mark the halfmass radii of the original disks.

In the dissipationless simulation, most of the stars within the half-mass radius become box orbits in the final remnant. When a dissipative component is added (second and third columns), the set of stars that became box orbits in the collisionless case instead form a population of $z$-tube orbits with largely canceling rotation, especially in disk 2 . It is apparent that this cancellation does not arise primarily from the ICs, since it occurs for stars in the same disk. Note that the spatial boundary of the canceling $z$-tube population changes little between $15 \%$ and $30 \%$ gas, suggesting that the boundary is set by a bar formation criterion that determines which orbits "would have been" boxes (e.g., Miller \& Smith 1979; Norman \& Silk 1983; Barnes \& Hernquist 1996; Bournaud et al. 2005a; MacMillan et al. 2006; Hopkins et al. 2009b), rather than by the direct gravity of the dissipative component. The box population might be fully transformed into $z$-tubes so long as a critical gas mass is exceeded. It should also be noted that the efficiency with which the gas is torqued inward decreases with increasing $f_{\text {gas }}$, so the CMC mass comprises a smaller fraction of the initial gas mass in higher- $f_{\text {gas }}$ remnants (see Figure 7 of Hopkins et al. 2009b).

Stars outside the half-mass radius in disk 1 generally become $z$-tube orbits in the remnant, with a higher degree of streaming than in the inner parts. The fate of these stars is relatively insensitive to $f_{\text {gas }}$, though some box orbits from this region in the dissipationless remnant do become $z$-tubes in the simulations with gas (compare the top right and top middle panels). The stars originating in the outer part of disk 2 end up in a uniformly streaming $x$-tube population, irrespective of $f_{\text {gas }}$. Their streaming about the long axis of the remnant can be explained simply in terms of the merger geometry-see, e.g., Figure 3 of Novak et al. (2006). The long axis lies in the merger plane, so stars on nearly circular orbits in the outskirts of an inclined disk start out with large tangential velocity components about this axis, and are therefore liable to occupy the $x$-tube "start space" (e.g., Schwarzschild 1979, 1993). The uniform rotation of these orbits in the final remnant suggests that they do not cross orbital boundaries during the merger; their angular momentum is retained from the initial spin of the inclined disk, and is a direct signature of the dynamically cold nature of the progenitor galaxy. Figure 7 provides a good example of how orbital classification can isolate sets of stars with similar physical histories.

So far we have focused the discussion on individual remnants; Figure 8 shows the dependence of the orbital structure on $f_{\text {gas }}$, averaged over all eight merger orbits. We compute the structural parameters on $\sim 1 R_{e}$ scales since this radius has been the main focus of triaxial dynamical modeling efforts to date (e.g., van de Ven et al. 2008; van den Bosch et al. 2008; de Lorenzi et al. 2006; van den Bosch 2008), and defer further discussion of the outer orbital structure to Section 3.4.

As expected from the preceding discussion, the $z$-tube population increases, the mass in box orbits decreases, and the shapes become rounder and more oblate with increasing $f_{\text {gas }}$. This transformation occurs primarily between $0 \%$ and $15 \%$ gas. At higher 


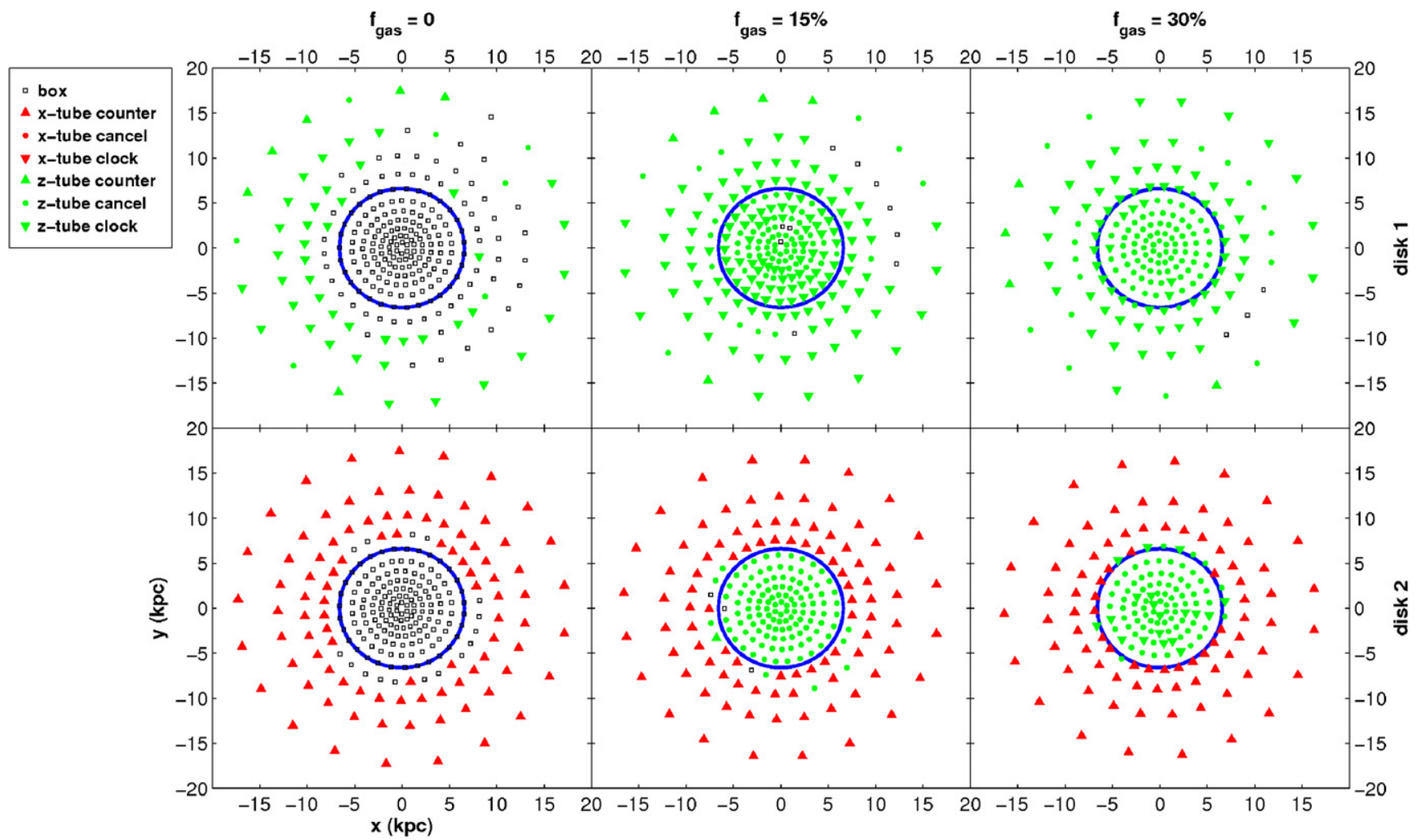

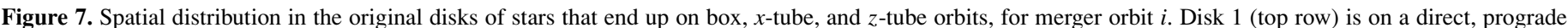

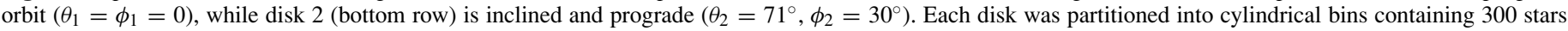

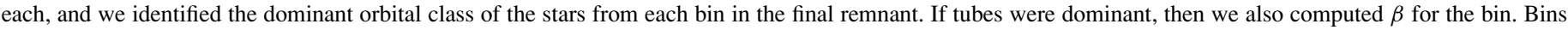

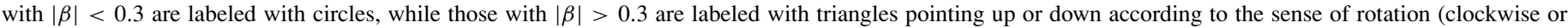

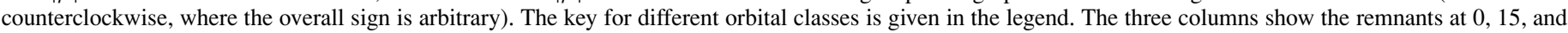
$30 \%$ gas. Only old stars are included (the gas particles in the initial disks are excluded). The blue circles mark the half-mass radii of the original disks.

(A color version of this figure is available in the online journal.)

gas fractions the average shapes, box, and $z$-tube fractions level off, consistent with the "critical mass" interpretation of the box orbit suppression. The apparent break in the trend at $30 \%$ gas arises because these remnants are very round. In a remnant that is very nearly spherical, the distinction between the two classes of tubes is blurred, and the triaxiality parameter and orientation vector are ill-defined (see e.g., remnant $m$ in Figure 19). The $40 \%$ gas remnants are slightly more flattened than those at $30 \%$ gas, presumably owing to the rotation of the stronger embedded disks. The stellar component is slightly more flattened than the mass including the DM, particularly at low $f_{\text {gas }}$. There is a mild downward trend in the population of $x$-tube orbits with $f_{\text {gas }}$ between zero and $15 \%$ as the shape of the potential becomes more oblate, but the round potentials at higher gas fractions are slightly more amenable to $x$-tubes.

At low $f_{\text {gas }}$, the $x$-tube orbits tend to stream far more than the $z$-tube orbits. This trend reverses at the highest gas fractions owing to the rapid rotation of the embedded disks, and the direct modification of the gravitational potential by the large CMC, which may blur the lines between $x$-tube and $z$-tube orbits as the potential becomes very round. The streaming of the $x$-tube orbits might also fall off because of the figure rotation; the long axis no longer necessarily lies in the merger plane, so more stars may be randomly scattered onto tangential orbits about the long axis. The exact reason for this trend in the $x$-tube rotation needs further investigation. The cancellation of the $z$-tube rotation is most complete around $10 \%$ gas, perhaps because the stronger CMC more efficiently disrupts box orbits than at lower $f_{\text {gas }}$, while at higher $f_{\text {gas }}$ the dissipation begins to play a direct role.
In the high- $f_{\text {gas }}$ remnants, the fraction of box orbits often turns upward in the very center. This effect is partially hidden in, e.g., Figure 20, since we started the profiles at the 15th percentile in binding energy. It can be naturally explained since only the innermost component (the CMC itself) contains no smaller CMC, and so can support stable box orbits. The violent relaxation during the final merger of the two cores provides a natural setting for the onset of triaxiality. However, the box population computed within the central starburst is expected to be highly sensitive to numerical effects, including artificial flattening of the core density profile owing to the gravitational softening, and imprecise centering in the orbital classification, so we chose to omit the very innermost scales from the structural profiles.

The remnants do not typically show large orientation twists within the 50th percentile in binding energy, or $\sim 1.5 R_{e}$ (excluding the KDCs, since the shape may not be well-resolved on such small scales). The low $f_{\text {gas }}$ remnants typically have large kinematic misalignments, while the angular momentum of the $40 \%$ gas remnants is well aligned with the short axis of the potential.

Profiles of the orbital structure, shape, and orientation of all 48 dissipative remnants (5\%-40\% gas) are shown in Figures 15 to 20 . We have outlined the main trends with $f_{\text {gas }}$ in this section, but there is substantial variation in the structural transformations from one merger orbit to the next. Some of the remnants $(l$ and $p$ ) are already transformed into oblate, $z$-tube-dominated systems at $5 \%$ gas, while others (e.g., $i$ and $k$ ) are still boxdominated and substantially prolate at this gas fraction. Remnant 


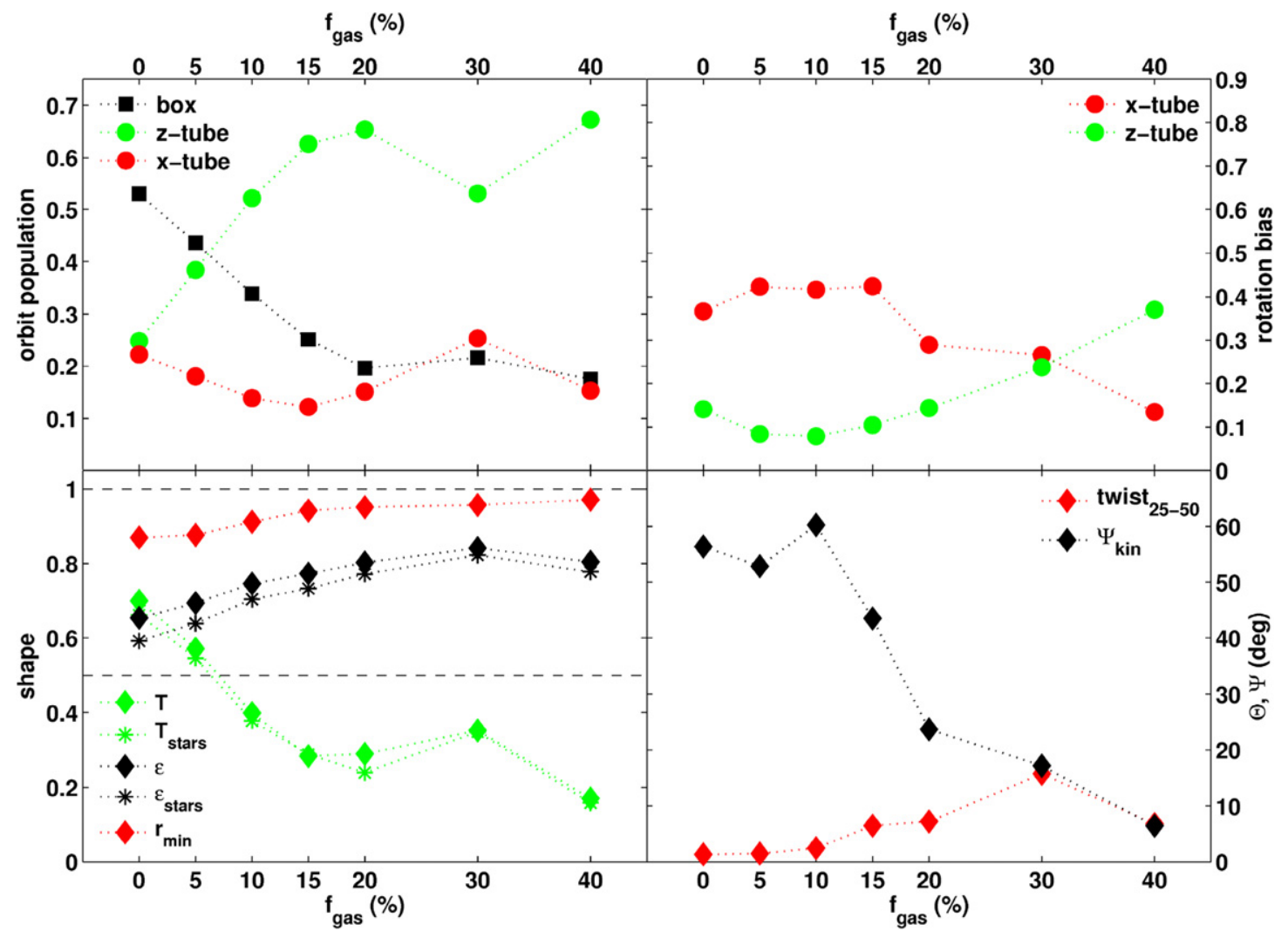

Figure 8. Inner structure $\left(\leqslant 1 R_{e}\right)$, averaged over eight merger orbits. Upper left: fraction of the stellar mass in each orbital class vs. $f_{\text {gas }}$, for the 25 th-45th percentile in binding energy $\left(\sim 0.5-1.3 R_{e}\right)$. Upper right: rotation bias $(\beta)$ of the $x$-tube and $z$-tube orbits, as defined in Equation (1). Lower left: intrinsic shape of the $40 \%$ most bound particles. The filled diamonds are for all matter (including DM particles), while the asterisks are for stars only. Lower right: intrinsic orientation twist between the 25th and 50th percentile in binding energy, and kinematic misalignment $\left(\Psi_{\text {kin }}\right)$ for the $40 \%$ most bound particles. $\Psi_{\text {kin }}$ is defined as in Figure 3. The orientation twist is computed as $\max \left(\theta_{i j}\right), i_{25}<(i, j)<i_{50}$, the maximum angle between the symmetry axes computed within any two cumulative energy bins between the 25 th and 50th percentiles.

(A color version of this figure is available in the online journal.)

$o$ is oblate at $0 \%$ gas and becomes prolate at $5 \%$ gas. A small gas component transforms remnant $j$ from a nearly pure prolate spheroid dominated by $x$-tube orbits, to a triaxial system with a large $z$-tube population. As the CMC alters the shape of the potential, both directly and indirectly through its interactions with the stars, the phase space boundaries between the orbital classes move. This shifting of the orbital boundaries can induce further changes in the potential, leading to a cascade effect. The causes of the more complex orbital transformations in some of the individual remnants are an interesting topic for future study.

\subsection{Kinematically Distinct Cores and Embedded Disks}

The 15\%-40\% gas remnants often contain a cold $z$-tube population that appears as a KDC or embedded disk in $\sim 1 R_{e}$ velocity maps, similar to the sub-components observed in some of the SAURON galaxies (Krajnovic et al. 2008).

This component typically appears as a KDC in the 15\%-20\% gas remnants, with a large kinematic twist relative to the stars around $1 R_{e}$. Figure 9 depicts five selected KDC systems, from a variety of different remnants and viewing angles. $1 R_{e}$ velocity maps are presented in the top row, while the bottom row shows the corresponding maps of $h_{3}$ to establish the disk-like character of the KDCs. In some cases there are also large photometric twists on the KDC scale, as in Column 4 of Figure 9. The KDCs are visible from nearly all viewing angles in the $\sim 50 \%$ of the remnants that have them (see Figures 17-18).

The outer parts of the KDC remnants show a variety of different types of kinematics, depending of the remnant and projec- tion-some show no rotation (e.g., Column 1 of Figure 9), some minor-axis or oblique rotation (Columns 2 and 3), and some a significant amount of major-axis rotation either aligned with or counter to the KDC (but always less disk-like than the KDC; e.g., Columns 2 and 4). $h_{3}$ and $v$ are always tightly anticorrelated within the KDC, but may have a positive (Columns 2 and 5) or weaker negative (Column 4) correlation outside the core. Some of the remnants show multiple kinematically distinct parts, e.g., remnant $i$ at $20 \%$ gas displays minor-axis rotation with $h_{3}$ and $v$ correlated, and major-axis rotation counter to the KDC, with $h_{3}$ and $v$ anticorrelated, at the outer edge of the map.

The cores of the KDC remnants are not nearly as distinct in their underlying orbital structure as they are in their kinematics, as van den Bosch et al. (2008) observed in the dynamical modeling of NGC 4365. This is because the mass of the streaming population producing the $\mathrm{KDC}$ is generally small, and the KDCs consist of an oblate $z$-tube population on top of a system already oblate and dominated by $z$-tube orbits because of the strong CMC. Figure 10 illustrates this point by showing the orbital breakdown of the LOSVDs at three different locations in the KDC remnant depicted in the second column of Figure 9: one within the $\mathrm{KDC}$ (point $\mathrm{A}$ ), one along the major axis outside the $\mathrm{KDC}$, in a region showing no rotation (point $\mathrm{B}$ ), and one near $1 R_{e}$ along the minor axis, in the part dominated by minor-axis rotation (point $\mathrm{C}$ ). The orbital population at all three locations is (perhaps surprisingly) comprised of roughly the same mixture of $z$-tube, $x$-tube, and box orbits, with $z$-tube orbits always in the majority. The very different appearance of the three regions 


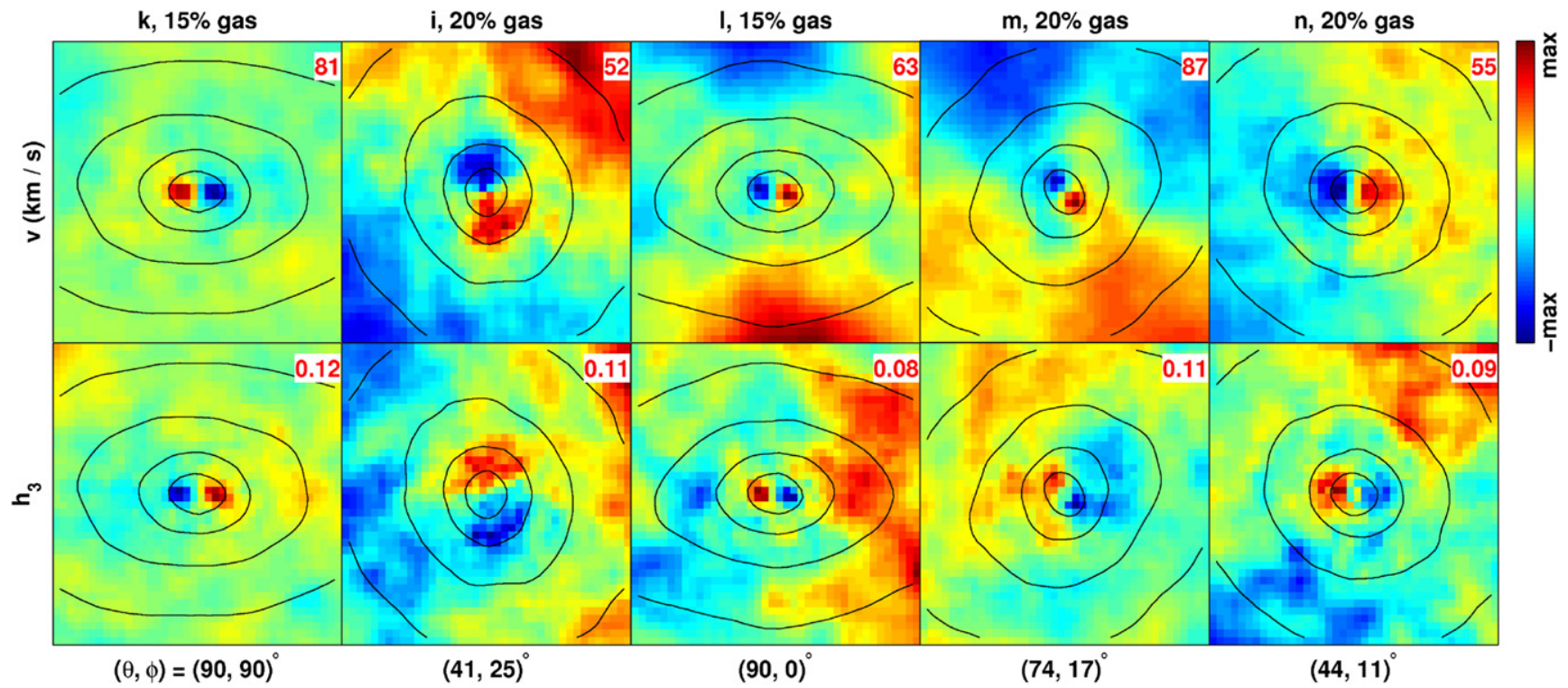

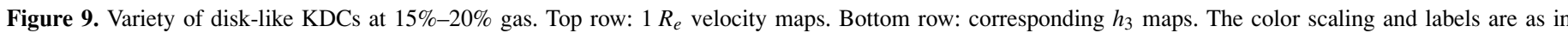
previous figures. Each column is labeled at the top with the merger orbit and gas fraction, and at the bottom with the LOS. (A color version of this figure is available in the online journal.)

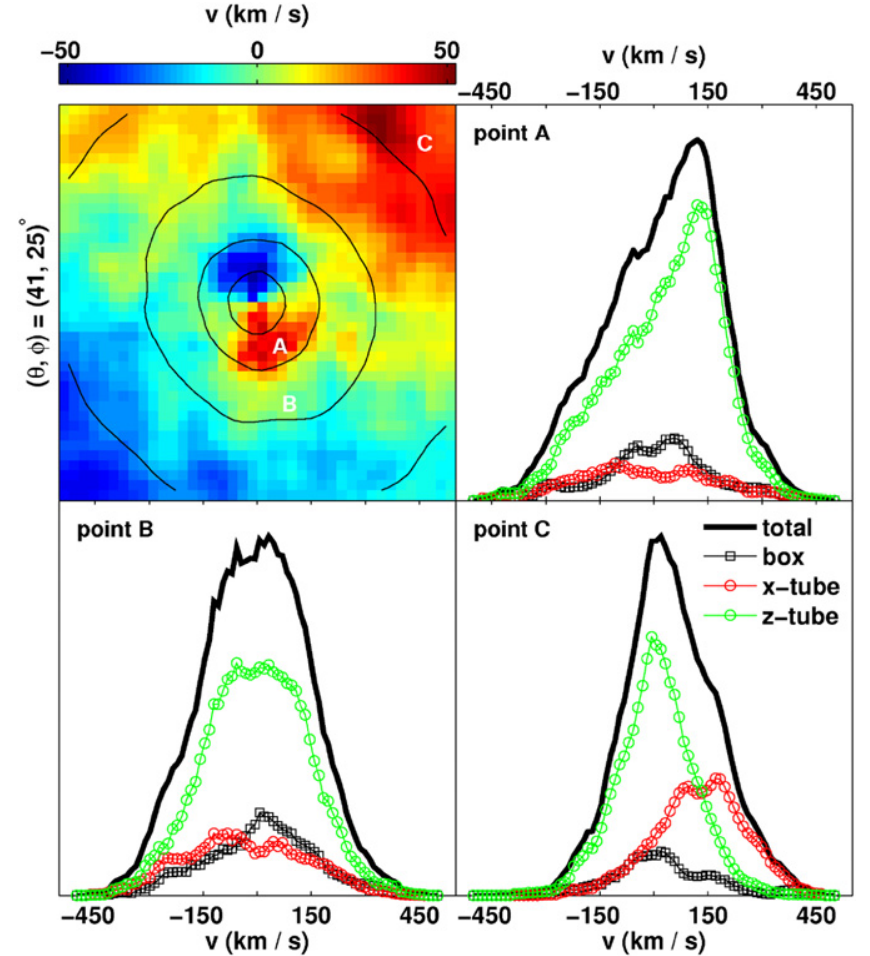

Figure 10. Orbital structure of a typical KDC remnant. Upper left: velocity map of remnant $i, 20 \%$ gas, viewed in an oblique projection. Upper right: LOSVD at location A, near the major axis within the KDC. Lower left: LOSVD at location $\mathrm{B}$, near the major axis outside the KDC. Lower right: LOSVD at location C, near the minor axis. Color scaling and labels are as in previous figures.

(A color version of this figure is available in the online journal.)

in the kinematic map owes to large differences in the degree of orbital streaming - at point A, the z-tubes are highly streaming, the $x$-tubes are highly streaming at point $\mathrm{C}$, and at point $\mathrm{B}$, both classes of tubes are distributed symmetrically about $v=0$.

In the $30 \%-40 \%$ gas remnants, the scale of the disk component is typically large enough to fill most or all of the SAURONscale kinematic maps. The embedded disk in these remnants is

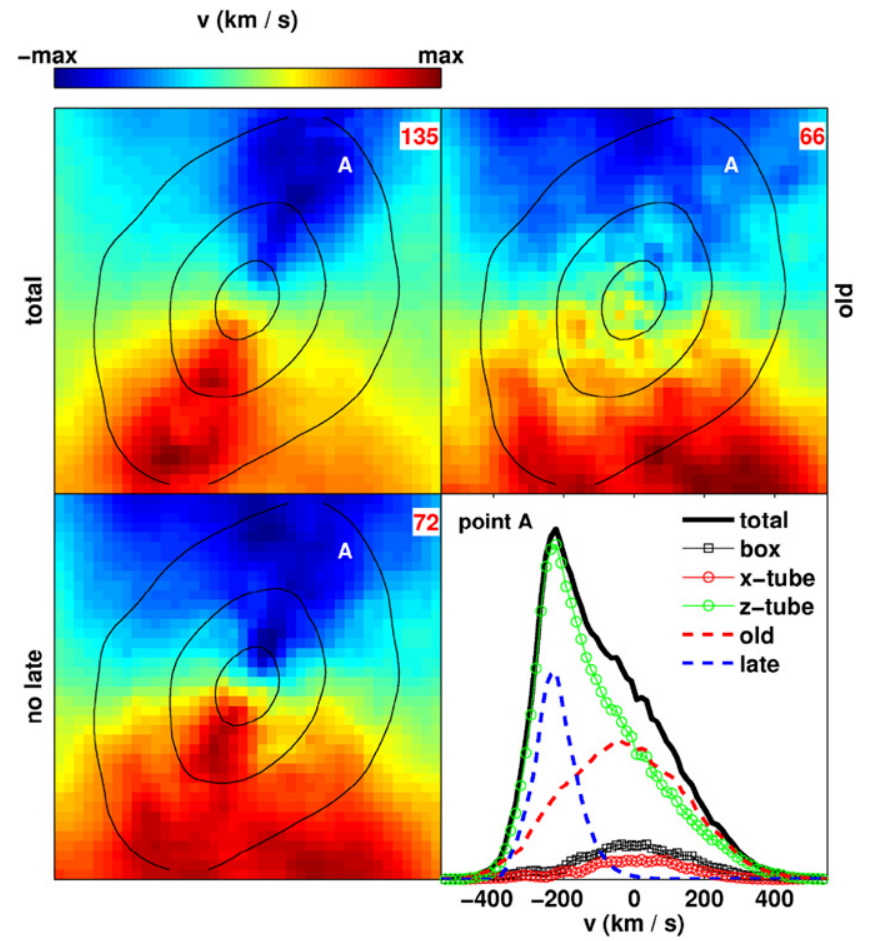

Figure 11. Embedded disk formed by dissipation. Upper left: velocity map of remnant $i, 40 \%$ gas, viewed at $(\theta, \phi)=\left(72^{\circ}, 77^{\circ}\right)$. Upper right: same map with the new stars, formed from gas during the merger, removed. Lower left: same map with only the $15 \%$ of the new stars that formed last removed. Lower right: LOSVD at location A, broken down by orbital class and stellar age. Color scaling and labels are as in previous figures.

(A color version of this figure is available in the online journal.)

typically quite cold, because the disk stars form in a slow trickle extending over $\sim 2$ Gyr after the merger (Robertson et al. 2006a; Hoffman 2007) and are therefore subject to very little violent relaxation. Figure 11 shows remnant $i$ at $40 \%$ gas, which has one of the strongest embedded disks in our sample, at a viewing angle about $30^{\circ}$ from edge on. The contribution from old stars, present as collisionless particles in the initial disks, is shown 


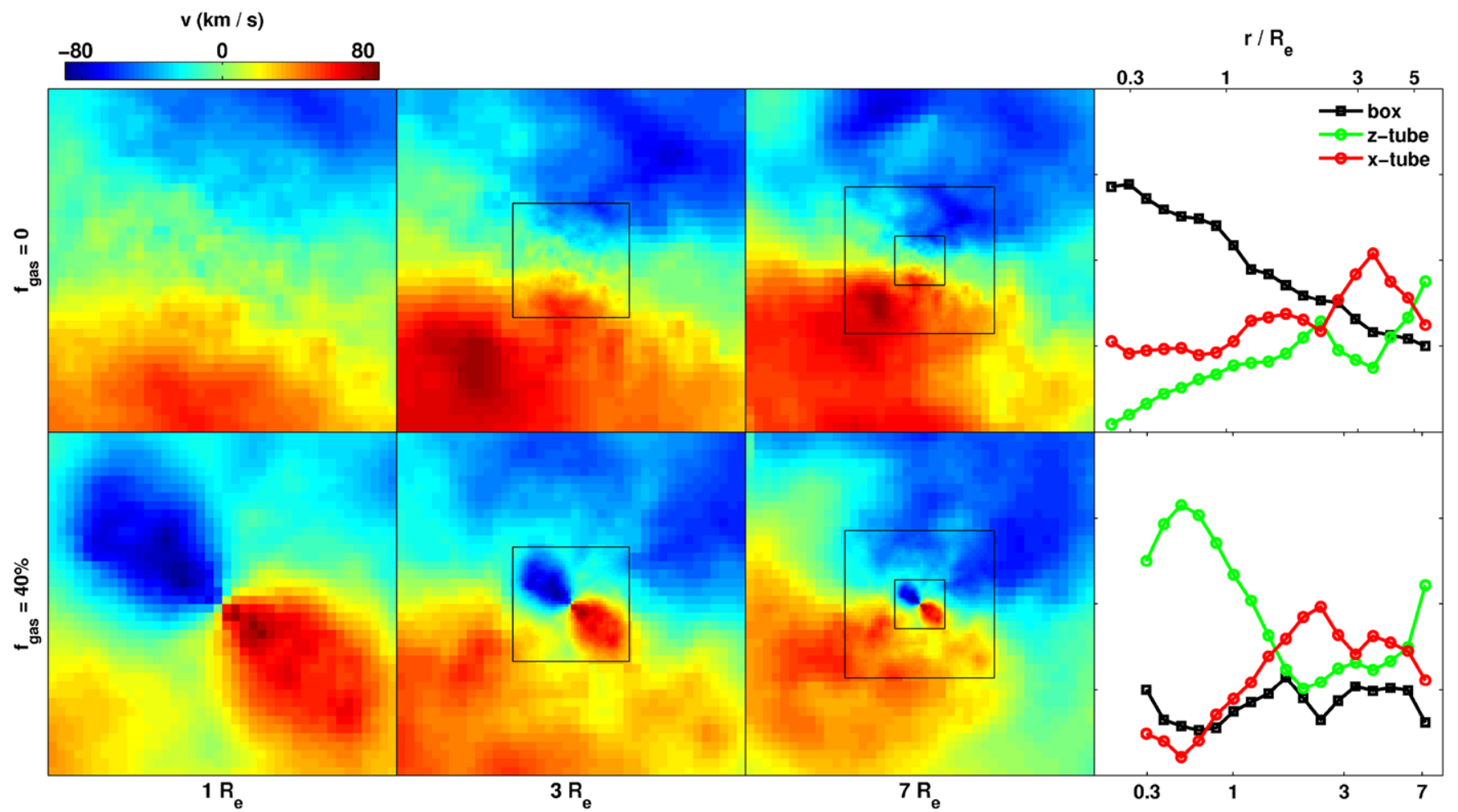

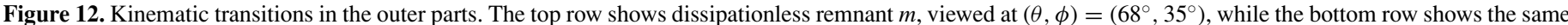

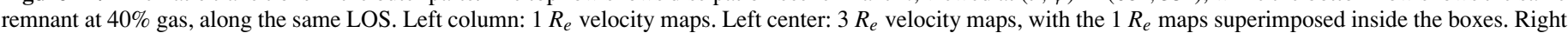

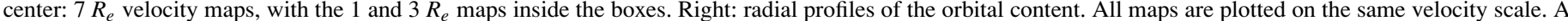
Gaussian (rather than $\mathrm{GH}$ ) fitting was used in this figure because of the noisy LOSVDs at large radii.

(A color version of this figure is available in the online journal.)

in the upper right panel. When only old stars are included, the maximum velocity on the map drops from 135 to $66 \mathrm{~km} \mathrm{~s}^{-1}$, and the rotation is no longer well aligned with the major axis. The old stars do still display more major-axis rotation than most remnants at low $f_{\text {gas }}$, since they also contract and spin up in response to the rapid condensation of the gas (e.g., Eggen et al. 1962; Blumenthal et al. 1986; Gnedin et al. 2004; Villalobos et al. 2009).

The lower right panel shows what is left when only $15 \%$ of the new stars that formed the latest $(\sim 5 \%$ of the total stellar mass) are removed. The maximum velocity still plummets to $72 \mathrm{~km} \mathrm{~s}^{-1}$, and what remains appears similar to a KDC system, since the most recently formed stars dominate the outer portions of the embedded disk. The contributions of old and recently formed stars to a typical LOSVD near $1 R_{e}$ in the embedded disk is shown in the lower right panel. The stars that formed late have a much smaller velocity dispersion, and when superimposed on the hot old population they yield a velocity distribution strongly skewed opposite the mean velocity (see also Hoffman et al. 2009).

\subsection{Outer Structure}

We have shown that the outer parts of the remnants (outside $\sim 1.5 R_{e}$ ) are less relaxed than the inner parts, and are largely unaffected by the gas content of the disks (see Figures 5 and 7). While kinematic maps of gas-rich remnants are dominated by dissipational rotation on $1 R_{e}$ scales, their kinematics farther out reflects the "dry" part of the merger remnant that is left essentially untouched by the gas, resulting in large kinematic twists between around 1 and $3 R_{e}$.

This point is illustrated clearly in Figure 12, which compares velocity maps of remnant $m$ at $0 \%$ and $40 \%$ gas on three different scales, 1,3 , and $7 R_{e}$, in the same oblique projection. Profiles of the orbital populations are reproduced in the right-hand panel for comparison. On $1 R_{e}$ scales the dissipationless and gas-rich remnants look entirely different, the former being a minoraxis rotator while the latter is dominated by rapid disk-like rotation. However, when we zoom out to $3 R_{e}$, the similarity in their outer structure becomes apparent. The gas has thoroughly transformed the inner part of the $40 \%$ gas remnant, while the outer parts are left as they were in the dissipationless case. The same observation can be made from the orbital profiles- the orbital populations outside $\sim 1.5 R_{e}$ are similar in the $0 \%$ and $40 \%$ gas remnants, but are radically different within $1 R_{e}$, where the dissipationless remnant is comprised mostly of box orbits and the $40 \%$ gas remnant is dominated by $z$-tubes. The similarity in the outer kinematic maps is even more apparent on $7 R_{e}$ scales.

Sharp kinematic and orbital transitions such as those in Figure 12 will be clearly observable in new surveys such as SMEAGOL, probing the stellar orbital structure out to $\sim 3 R_{e}$ scales (Proctor et al. 2009; Foster et al. 2009), or the PN.S survey, probing the kinematics out to $\sim 7 R_{e}$ scales using PNe as tracers (Douglas et al. 2002; Romanowsky et al. 2003; Coccato et al. 2009). Predictions for the statistics of kinematic twists and abrupt changes in the rotation parameter, $\lambda_{R}$ (Emsellem et al. 2007), in such surveys will be further discussed and quantified in L. Hoffman et al. (2009a, in preparation).

The intrinsic outer structure of the remnants is summarized in Figure 13, which is the same as Figure 8 but for the structure between $\sim 3$ and $7 R_{e}$. The orbital populations, shapes, and orbital streaming are far less sensitive to $f_{\text {gas }}$ than in the inner parts. The outer stellar population is dominated by tube orbits, as expected given the steep effective density profile at these 


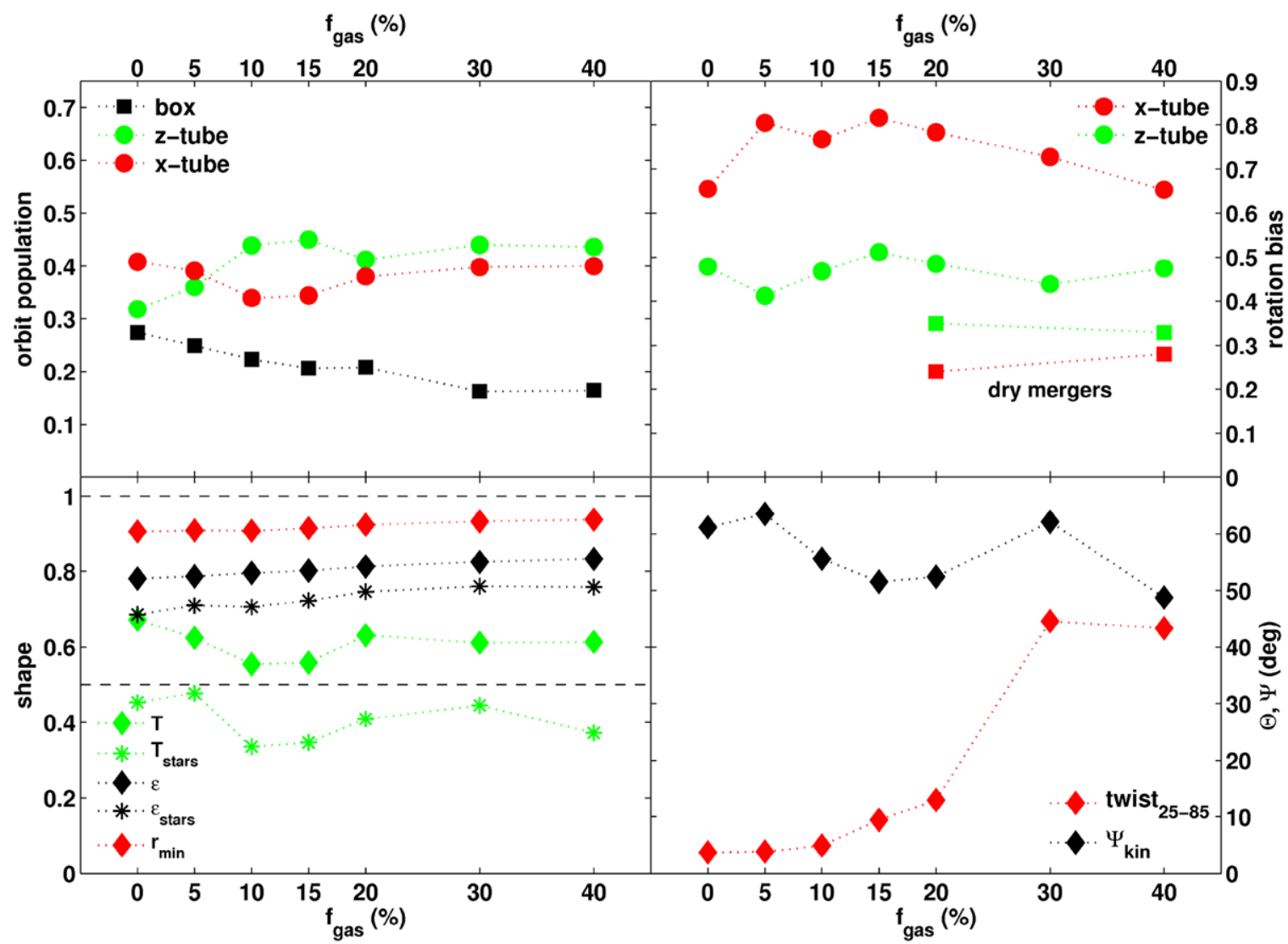

Figure 13. Outer structure $\left(\sim 3-7 R_{e}\right)$, averaged over eight merger orbits. Same as Figure 8 , except that the orbital structure is averaged over the 75 th-95th percentiles in binding energy, and the shapes and kinematic misalignment are evaluated within the 80th percentile. The intrinsic orientation twist is computed as max $\left(\theta_{i j}\right)$, with $i$ and $j$ running from the 25 th to the 85 th percentile in binding energy $\left(\sim 0.5-5 R_{e}\right)$. On the tube rotation plot (upper right panel), we have added data points for re-mergers of the $20 \%$ and $40 \%$ gas remnants (labeled as "dry" mergers-see the text).

(A color version of this figure is available in the online journal.)

radii, with a roughly even mix of $x$-tubes and $z$-tubes. The exchange between box and $z$-tube orbits with increasing $f_{\text {gas }}$ is still noticeable, though diminished. From visual inspection of some randomly selected orbits, box orbits in the halo tend to be more stochastic and spherical in shape (Clozel 2008), and we will further investigate the nature of the outer box orbits in future work.

Both classes of tubes have a higher rotation bias than in the inner parts. The $x$-tube orbits are especially highly streaming, which is a signature of the stars' dynamically cold origin. To highlight this point, we have also plotted the streaming fraction of the $x$-tube and $z$-tube orbits over the same range of relative binding energies for a series of re-mergers of the $20 \%$ and $40 \%$ gas remnants (see Hoffman et al. 2009), intended to represent "dry" mergers between elliptical galaxies that have already exhausted their gas in a previous major merger (e.g., van Dokkum 2005; Bell et al. 2006; Naab et al. 2006b; Kormendy et al. 2009). The streaming of the $z$-tubes is not that much different in the remnants of mergers between dynamically hot ellipticals than in the disk mergers, but the $x$-tube orbits show far less ordered rotation in the dry merger remnants. In a merger between hot systems, the random motions of the stars provide a source of initial angular momentum about the long axis, with no preferred sense of rotation. The uniform streaming of the outer $x$-tube orbits might be a tell-tale sign of a cold (late-type) progenitor in dynamical models of observed systems.

The shapes of the matter distribution between 3 and $7 R_{e}$ are round ( $\epsilon \sim 0.8$ on average), and nearly maximally triaxial.
The stars are substantially more flattened $(\epsilon \sim 0.75)$ and oblate $(T \sim 0.4)$ than the matter as a whole (including DM). Large kinematic misalignments are common in the outer parts, producing large kinematic twists between $\sim 1$ and $3 R_{e}$ in the most gas-rich remnants, as shown previously in Figure 12. The $30 \%-40 \%$ gas remnants also typically have large intrinsic orientation twists between $\sim 1$ and $5 R_{e}$, owing to the rapid figure rotation of the inner component.

\section{SUMMARY AND CONCLUSIONS}

We have shown that a variety of observed kinematic structures can be accounted for just by varying the gas fraction in binary 1:1 mergers. The projected kinematics within $1 R_{e}$ tend to fall into one of four categories: (1) rapid disk-like rotation about the major axis, (2) a prominent, disk-like KDC with slow or minor-axis rotation farther out, (3) uniform slow rotation, with $v_{\max } \lesssim 40 \mathrm{~km} \mathrm{~s}^{-1}$, and (4) prominent minor axis rotation, with little or no rotation about the major axis. In Figure 14, we classify the eight remnants at each $f_{\text {gas }}$ into these four categories based on visual inspection of their velocity maps in projection along the $y$-axis (intrinsic variations far outweigh projection effects in the global appearance of the kinematic maps). The 0\%-10\% gas remnants are generally slowly rotating or dominated by minor-axis rotation, in agreement with the previous results of Naab et al. (2006a); Jesseit et al. (2007); Cox et al. (2006a). The $15 \%-20 \%$ gas remnants often look similar to the lower- $f_{\text {gas }}$ remnants but with disk-like KDCs at their centers, and bear an 


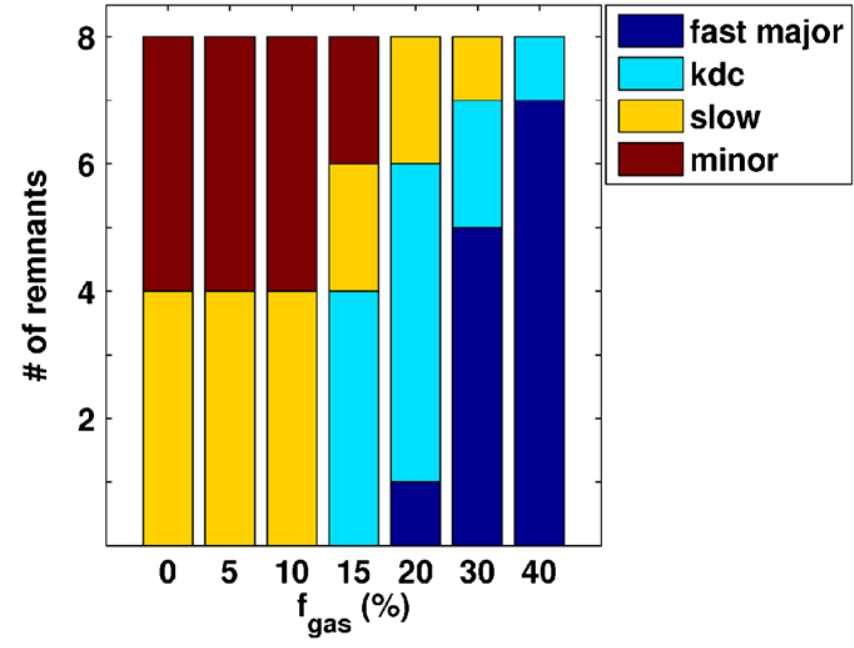

Figure 14. Classification of the remnant kinematics. The eight remnants of each gas fraction were categorized as (1) showing rapid major-axis rotation, (2) slowly rotating with a disk-like KDC, (3) slowly rotating throughout, or (4) showing rapid minor-axis rotation, based on visual inspections of their velocity maps (see the text).

(A color version of this figure is available in the online journal.)

interesting resemblance to observed galaxies such as NGC 4365 (Statler et al. 2004; van den Bosch et al. 2008; L. Hoffman et al. 2009b, in preparation), NGC 5813, and NGC 4458 (Emsellem et al. 2004; Cappellari et al. 2007; van den Bosch 2008).

The 30\%-40\% gas remnants generically show rapid, disklike rotation with $v / \sigma$ up to $\sim 1$ (see also Rix \& White 1990; Scorza \& Bender 1995; Robertson et al. 2006a; Krajnovic et al. 2008). None of them display the extreme rotation $(v / \sigma \sim 2)$ of the most rapid rotators in the SAURON sample, although other studies (Robertson et al. 2006a; Robertson \& Bullock 2008) have shown that $1: 1$ mergers between $60 \%$ and $80 \%$ gas disks can yield higher values of $v / \sigma$. High $v / \sigma$ values are always accompanied by high, anticorrelated $h_{3}$ values in our remnants, so the subset of SAURON rapid rotators with a shallow $h_{3}-v / \sigma$ relation may require a different formation mechanism.

The variety of features that can be produced with 1:1 mergers alone suggests that there are degeneracies in the formation scenarios leading to a given gross outcome. For instance cold embedded disks can arise either from gas-rich merging or from cosmological gas inflow and secular evolution (Naab \& Burkert 2001; Robertson et al. 2004; Dekel \& Birnboim 2006; Robertson et al. 2006a; Bournaud et al. 2007a; Robertson \& Bullock 2008; Elmegreen et al. 2008; Genzel et al. 2008; Shapiro et al. 2008; Dekel et al. 2009; Naab et al. 2007; Keres \& Hernquist 2009; Ceverino et al. 2010). Lower progenitor mass ratios ( 3-4:1 instead of 1:1 mergers) can produce remnants resembling many SAURON rapid rotators even in dissipationless simulations (Naab et al. 1999; Cretton et al. 2001; Naab \& Burkert 2003; Bournaud et al. 2004, 2005b; Burkert \& Naab 2005; Jesseit et al. 2007; Hopkins et al. 2009b; Jesseit et al. 2009; Johansson et al. 2009). Sequential minor mergers can produce slowly rotating ellipticals, as well as major mergers with low $f_{\text {gas }}$, that are in better agreement with observed round, isotropic, and featureless systems such as NGC 4486, NGC 4552, and NGC 5846 (Weil \& Hernquist 1994, 1995; Bournaud et al. 2007b; Cappellari et al. 2007; Burkert et al. 2008; Naab et al. 2009; GonzalezGarcia et al. 2009). These scenarios must be distinguished with more detailed structural and dynamical analysis (e.g., Naab et al. 2006b; Jesseit et al. 2007; Burkert et al. 2008; de Lorenzi et al. 2008; van den Bosch et al. 2008; Robertson \& Bullock 2008; Shapiro et al. 2008; Jeong et al. 2008; Hoffman et al. 2009; Thomas et al. 2009; Romanowsky et al. 2009b; Romano-Diaz et al. 2009).

Our orbital analysis suggests a rather simple picture of how the structure of the remnants arises. Non-axisymmetric torques in the merger trigger the formation of a bar-like structure with a large population of box orbits. When gas is present, the strong CMC formed through dissipation (e.g., Mihos \& Hernquist 1994a) converts the majority of the box population into $z$-tube orbits, which have nearly canceling rotation since box diffusion has no preferred direction.

Stars in inclined disks begin with a large polar angular momentum component $\left(j_{\theta}\right)$ that is retained throughout the merger, so they do not cross orbital boundaries. This produces a streaming $x$-tube population in the remnants, that is most pronounced at large radii. This picture of the $x$-tube population is supported by the facts that (1) co-planar mergers produce remnants almost entirely devoid of $x$-tube orbits, (2) the phase space available to $x$-tubes in the remnant potentials is generally underpopulated (Barnes 1992; Hoffman 2007), and (3) in some observed ellipticals the velocity ellipsoid is flattened along the polar axis (van den Bosch 2008).

In gas-rich merger remnants, there is also a pronounced streaming $z$-tube population on small scales owing to the gas that dissipates energy while retaining substantial angular momentum during the merger (Hopkins et al. 2009b). Outside a fairly well-defined boundary (see e.g., Figures 7 and 12), the orbital structure is largely unaffected by the gas. This boundary corresponds to the radius where box orbits cease to dominate in the dissipationless remnants, implying that these regions may be left relatively intact because they have never had a large population of radial orbits to convey information about the galactic center to their location. Time-dependent orbital classification and idealized evolving models are needed to verify this interpretation (e.g., L. Hoffman et al. 2009b, in preparation; Villalobos et al. 2009).

The characteristic orbital structure arising from this picture, and in particular the sharp kinematic transitions predicted between $\sim 1$ and $3 R_{e}$, should be readily observable with surveys in progress such as SMEAGOL. Some pronounced kinematic transitions have been observed around these radii in survey pilot studies (Proctor et al. 2009; Coccato et al. 2009), but more data will be needed to determine whether these transitions are of the same nature as those in the merger simulations. Note that some features, e.g., the uniform streaming of the $x$-tube orbits, can only be captured with dynamical modeling - substantial minoraxis rotation alone could be produced by either a dominant population of $x$-tubes with a small rotation bias, or a smaller, highly streaming population.

One of the challenges in setting up suites of galaxy merger simulations is the large number of important free parameters in the ICs, e.g., the absolute masses and mass ratio of the progenitors, the merger impact parameter, the initial gas and bulge fractions, and the initial scale radii of the stellar and gas components. In this paper, we analyzed a very restricted set of binary merger remnants-1:1 mergers between pure disks on fairly radial encounter orbits - and focused on a physical understanding of the effect of varying the gas fraction in this idealized case. The choice of pure exponential disks for our ICs maximizes the susceptibility to bar formation and outward angular momentum transport; we will explore the effect of initial 


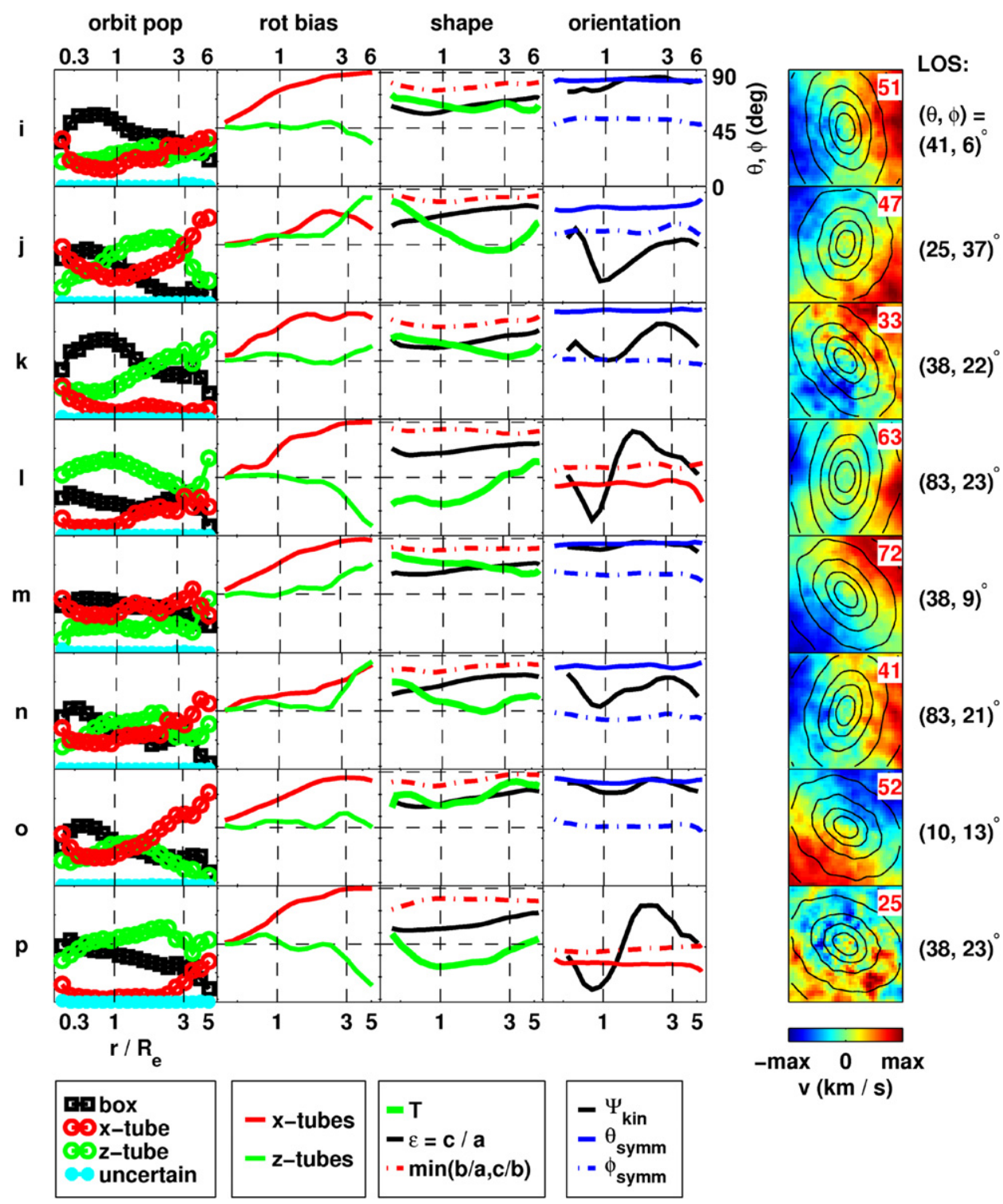

Figure 15. Intrinsic structure of the $5 \%$ gas remnants.

(A color version of this figure is available in the online journal.)

bulges in a future work (L. Hoffman et al. 2009a, in preparation). For simplicity and ease of comparison with previous studies (Cox et al. 2006a; Naab et al. 2006a), we also used the same scale length for the initial gas and stellar disks, although there is evidence that gas disks may be substantially more extended (e.g., Maller et al. 2001; Thilker et al. 2007). The mass in gas that collapses to the center to form the CMC is determined roughly by the total mass in the initial gas disks interior to the "corotation" radius (Hopkins et al. 2009b). Therefore, for a fixed gas fraction, a more extended gas disk may decrease the CMC mass and enhance the embedded disk in the remnant - there is a need for future simulations exploring this effect.

Although our simulations are idealized, our results illustrate how orbital analysis can isolate subsets of the stellar population with similar histories, and place intuitive constraints on galaxy formation mechanisms. In future work, we hope to extend this type of analysis to a broader range of merger parameters and more complex cosmological formation scenarios.

We thank Glenn van de Ven, Remco van den Bosch, Aaron Romanowsky, and Phil Hopkins for enlightening discussions, especially on relating our work to observations. We are also grateful to Bart Willems and Jaczek Braden for technical help. This work was supported in part by a Lindheimer Postdoctoral Fellowship at Northwestern University. Computations were performed on the Fugu computer cluster at Northwestern, funded by NSF MRI grant PHY-0619274 to Vicky Kalogera, and the Sauron cluster at the parallel computing center of the Institute for Theory and Computation at the HarvardSmithsonian Center for Astrophysics.

\section{APPENDIX}

In Figures 15-20, we present radial profiles of the orbital populations, intrinsic shapes, and orientations of all eight merger remnants with each initial gas fraction $(5 \%, 10 \%, 15 \%, 20 \%$, $30 \%$, and $40 \%$ ). The format of the figures is identical to that of Figure 4 in the body of the paper (intrinsic structure of the dissipationless remnants). The figures may be viewed one-at-atime to get a feel for the variation with merger orbit at fixed $f_{\text {gas }}$, or compared row-for-row to follow the evolution of the structure with $f_{\text {gas }}$ for a fixed merger orbit. 


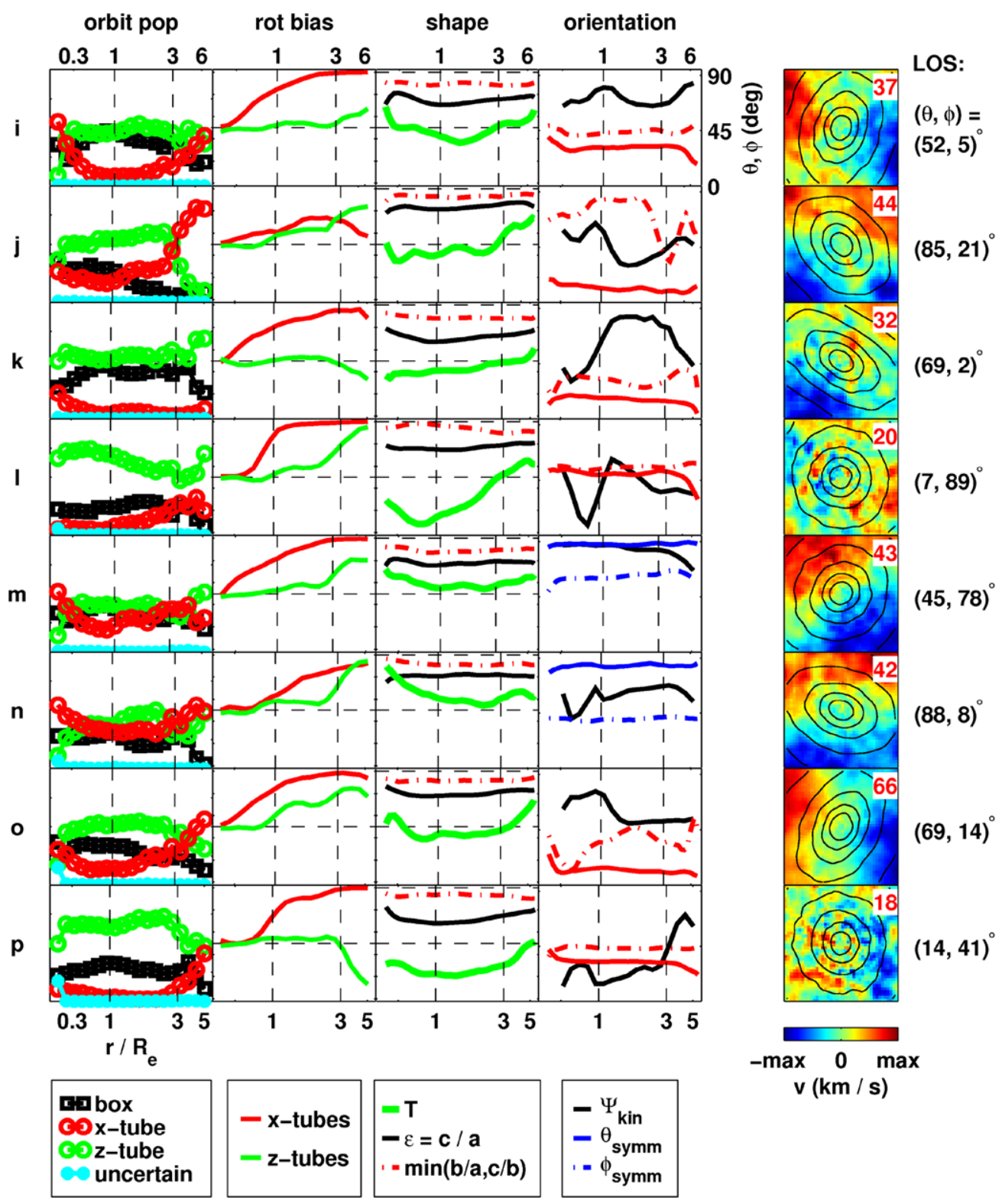

Figure 16. Intrinsic structure of the $10 \%$ gas remnants.

(A color version of this figure is available in the online journal.) 


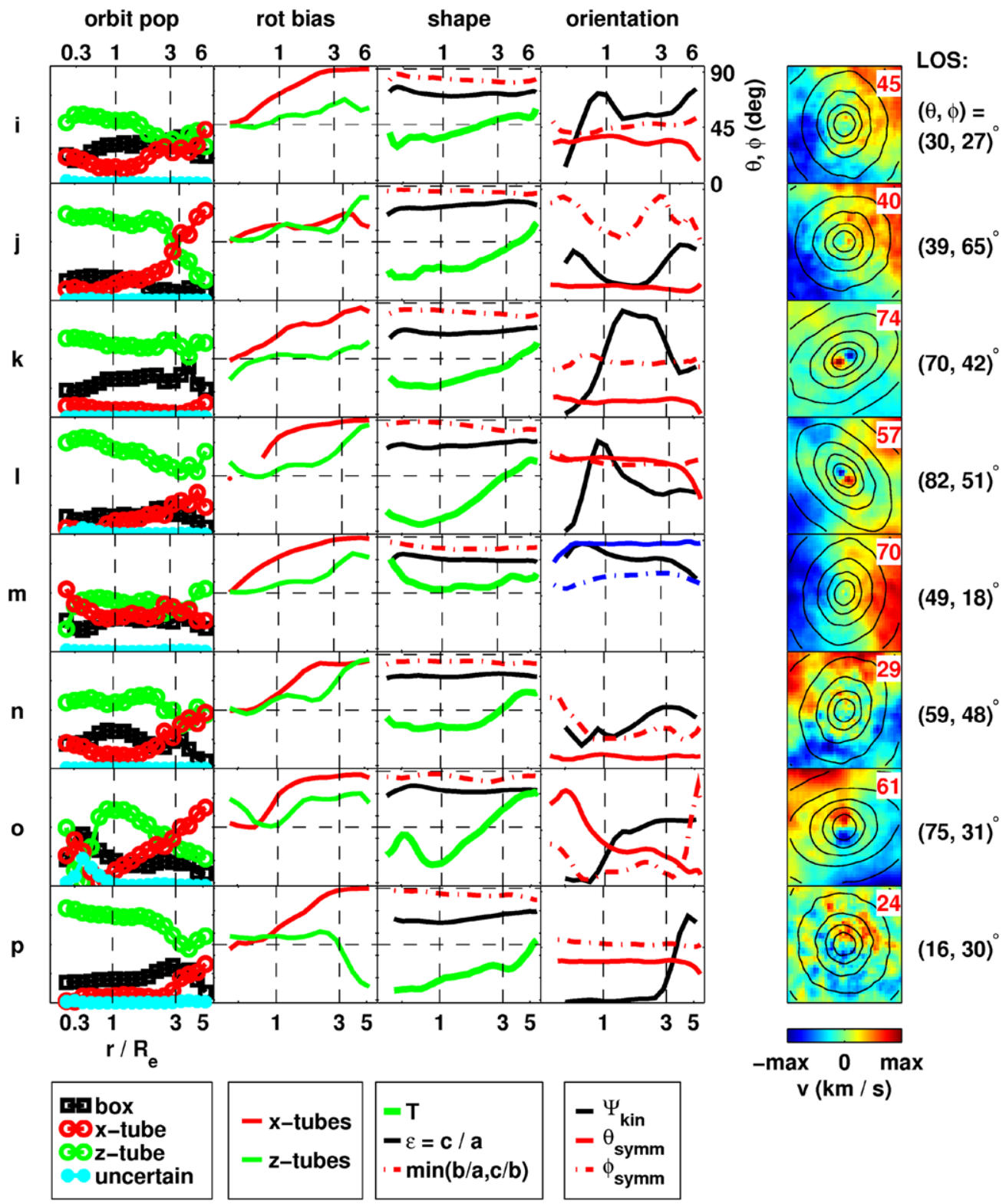

Figure 17. Intrinsic structure of the $15 \%$ gas remnants.

(A color version of this figure is available in the online journal.) 


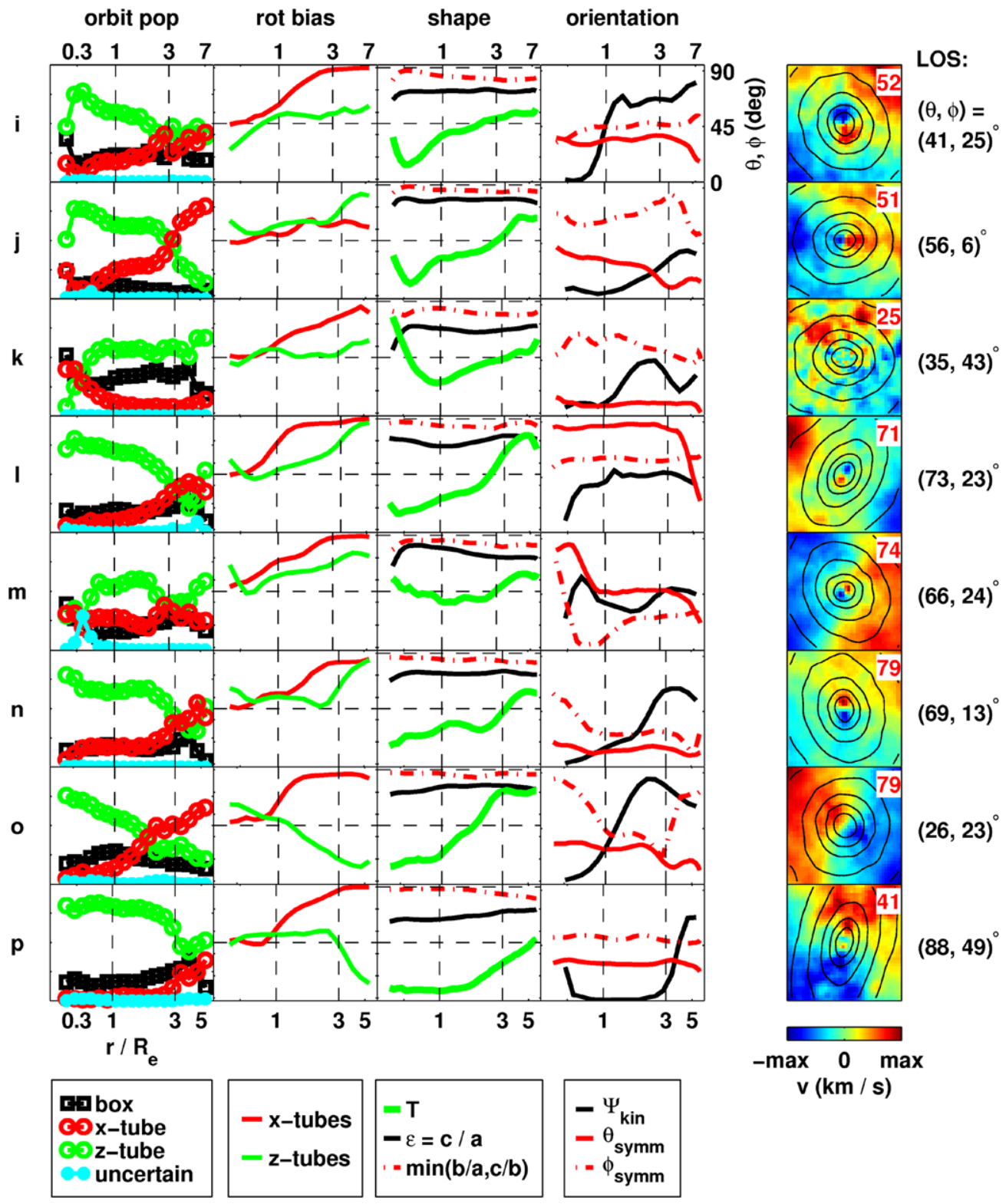

Figure 18. Intrinsic structure of the $20 \%$ gas remnants.

(A color version of this figure is available in the online journal.) 


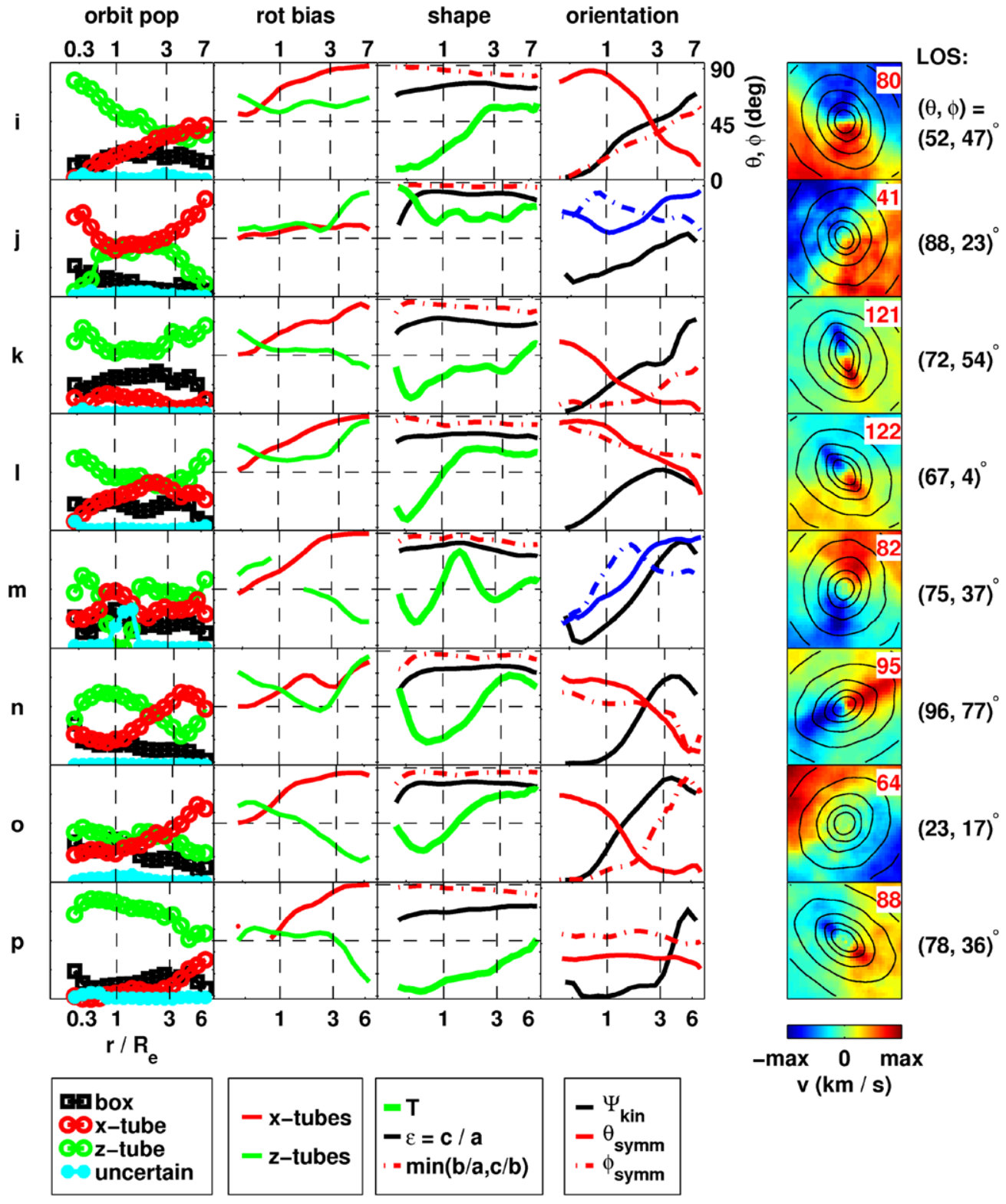

Figure 19. Intrinsic structure of the $30 \%$ gas remnants.

(A color version of this figure is available in the online journal.) 


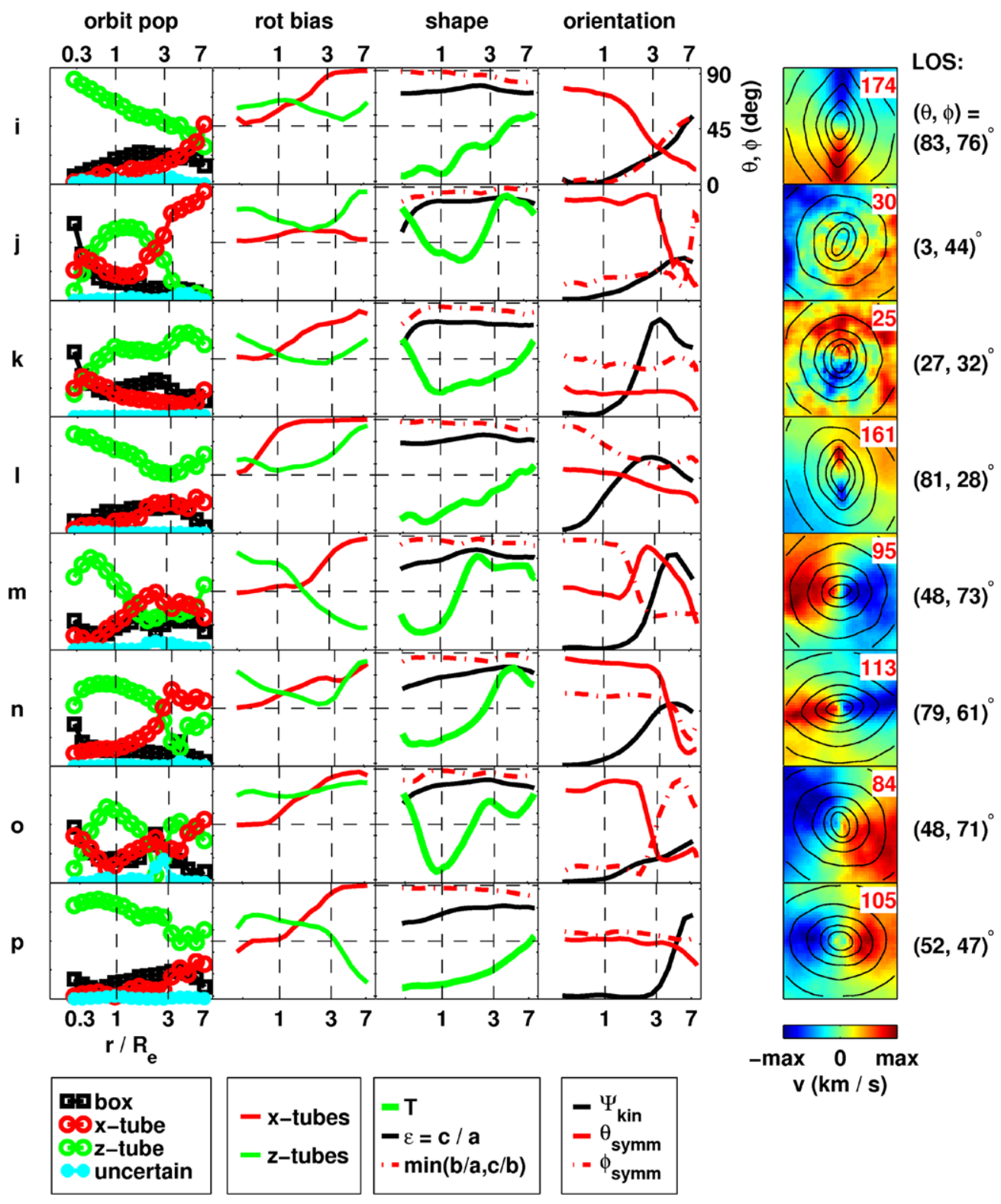

Figure 20. Intrinsic structure of the $40 \%$ gas remnants.

(A color version of this figure is available in the online journal.) 


\section{REFERENCES}

Aarseth, S. J., \& Binney, J. 1978, MNRAS, 185, 227

Abadi, M. G., Navarro, J. F., Fardal, M., Babul, A., \& Steinmetz, M. 2010, MNRAS, 407, 435

Aguilar, L. A., \& Merritt, D. 1990, ApJ, 354, 33

Alladin, S. M. 1965, ApJ, 141, 768

Arp, H. 1966, ApJS, 14, 1

Ashman, K. M., \& Zepf, S. E. 1992, ApJ, 384, 50

Bacon, R., et al. 1995, A\&AS, 113, 347

Bacon, R., et al. 2001, MNRAS, 326, 23

Barnes, J. E. 1988, ApJ, 331, 699

Barnes, J. E. 1992, ApJ, 393, 484

Barnes, J. E. 1998, in Galaxies: Interactions and Induced Star Formation, ed. R. C. Kennicutt et al. (Berlin: Springer), 275

Barnes, J. E., \& Hernquist, L. E. 1991, ApJ, 370, L65

Barnes, J. E., \& Hernquist, L. E. 1996, ApJ, 471, 115

Bell, E. F., et al. 2006, ApJ, 640, 241

Bellovary, J. M., Dalcanton, J. J., Babul, A., Quinn, T. R., Maas, R. W., Austin, C. G., Williams, L. L. R., \& Barnes, E. I. 2008, ApJ, 685, 739

Bendo, G. J., \& Barnes, J. E. 2000, MNRAS, 316, 315

Bender, R., Saglia, R. P., \& Gerhard, O. E. 1994, MNRAS, 269, 785

Bertschinger, E. 1985, ApJS, 58, 39

Best, P. N., von der Linden, A., Kauffmann, G., Heckman, T. M., \& Kaiser, C. R. 2007, MNRAS, 379, 894

Binney, J. 1985, MNRAS, 212, 767

Binney, J., \& Merrifield, M. 1998, Galactic Astronomy (Princeton, NJ: Princeton Univ. Press)

Binney, J., \& Spergel, D. 1982, ApJ, 252, 308

Binney, J., \& Spergel, D. 1984, MNRAS, 206, 159

Binney, J., \& Tremaine, S. 2008, Galactic Dynamics (Princeton, NJ: Princeton Univ. Press)

Blanc, G. A., Heiderman, A., Gebhardt, K., Evans, N. J., \& Adams, J. 2009, ApJ, 704,842

Block, D. L., Bournaud, F., Combes, F., Puerari, I., \& Buta, R. 2002, A\&A, 394, 35

Blumenthal, G. R., Faber, S. M., Flores, R., \& Primack, J. R. 1986, ApJ, 301, 27

Bond, J. R., Cole, S., Efstathiou, G., \& Kaiser, N. 1991, ApJ, 379, 440

Bondi, H. 1952, MNRAS, 112, 195

Bondi, H., \& Hoyle, F. 1944, MNRAS, 104, 273

Bournaud, F., Jog, C. J., \& Combes, F. 2004, A\&A, 418, 27

Bournaud, F., Combes, F., \& Semelin, B. 2005a, MNRAS, 364, 18

Bournaud, F., \& Elmegreen, B. G. 2009, ApJ, 694, L158

Bournaud, F., Elmegreen, B. G., \& Elmegreen, D. M. 2007a, ApJ, 670, 237

Bournaud, F., Jog, C. J., \& Combes, F. 2005b, A\&A, 437, 69

Bournaud, F., Jog, C. J., \& Combes, F. 2007b, A\&A, 476, 1179

Bulirsch, R., \& Stoer, J. 1966, Numer. Math., 8, 1

Bullock, J. S., Dekel, A., Kolatt, T. S., Kravtsov, A. V., Klypin, A. A., Porciani, C., \& Primack, J. R. 2001a, ApJ, 555, 240

Bullock, J. S., Kolatt, T. S., Sigad, Y., Somerville, R. S., Kravtsov, A. V., Klypin, A. A., Primack, J. R., \& Dekel, A. 2001b, MNRAS, 321, 559

Burkert, A., \& Naab, T. 2005, MNRAS, 363, 597

Burkert, A., Naab, T., Johansson, P. H., \& Jesseit, R. 2008, ApJ, 685, 897

Cappellari, M., et al. 2007, MNRAS, 379, 418

Carlberg, R. G. 1986, ApJ, 310, 593

Carpintero, D. D., \& Aguilar, L. A. 1998, MNRAS, 298, 1

Ceverino, D., Dekel, A., \& Bournaud, F. 2010, MNRAS, 404, 215

Ceverino, D., \& Klypin, A. 2009, ApJ, 695, 292

Chaname, J., Kleyna, J., \& van der Marel, R. 2008, ApJ, 682, 841

Chandraskehar, S. 1943, ApJ, 97, 255

Clozel, G. 2008, Bachelor's thesis, Northwestern Univ.

Clutton-Brock, M. 1972, Ap\&SS, 16, 101

Clutton-Brock, M. 1973, Ap\&SS, 23, 55

Coccato, L., et al. 2009, MNRAS, 394, 1249

Cole, S., Lacey, C. G., Baugh, C. M., \& Frenk, C. S. 2000, MNRAS, 319, 168

Collett, J. L., Dutta, S. N., \& Evans, N. W. 1997, MNRAS, 285, 49

Conroy, C., \& Wechsler, R. H. 2009, ApJ, 696, 620

Contopoulos, G. 1960, Z. Astrophys., 49, 273

Contopoulos, G. 1963a, AJ, 68, 1

Contopoulos, G. 1963b, ApJ, 138, 1297

Contopoulos, G., \& Mertzanides, C. 1977, A\&A, 61, 477

Cox, T. J., Dutta, S. N., Di Matteo, T., Hernquist, L., Hopkins, P. F., Robertson, B., \& Springel, V. 2006a, ApJ, 650, 791

Cox, T. J., Jonsson, P., Primack, J. R., \& Somerville, R. S. 2006b, MNRAS, 373,1013

Crane, P., et al. 1993, AJ, 106, 1371
Cretton, N., Naab, T., Rix, H.-W., \& Burkert, A. 2001, ApJ, 554, 291

Croton, D. J., et al. 2006, MNRAS, 365, 11

Daddi, E., et al. 2010, ApJ, 713, 686

Davé, R., Hernquist, L., Katz, N., \& Weinberg, D. H. 1999, ApJ, 511, 521

Davies, R. L., et al. 2001, ApJ, 548, 33

D’Onghia, E., Besla, G., Cox, T. J., \& Hernquist, L. 2009, Nature, 460, 605

de Lorenzi, F., Debattista, V. P., Gerhard, O., \& Sambhus, N. 2006, MNRAS, 376,71

de Lorenzi, F., Gerhard, O., Saglia, R. P., Sambhus, N., Debattista, V. P., Pannella, M., \& Mendez, R. H. 2008, MNRAS, 385, 1729

de Lorenzi, F., et al. 2009, MNRAS, 395, 76

De Lucia, G., Springel, V., White, S. D. M., Croton, D., \& Kauffmann, G. 2006, MNRAS, 366, 499

de Zeeuw, P. T. 1985, MNRAS, 216, 273

de Zeeuw, P. T., \& Lynden-Bell, D. 1985, MNRAS, 215, 713

Debattista, V. P., Moore, B., Quinn, T., Kazantzidis, S., Maas, R., Mayer, L. Read, J., \& Stadel, J. 2008, ApJ, 681, 1076

Dekel, A., \& Birnboim, Y. 2006, MNRAS, 368, 2

Dekel, A., Sari, R., \& Ceverino, D. 2009, ApJ, 703, 785

Di Matteo, T., Springel, V., \& Hernquist, L. 2005, Nature, 433, 604

Djorgovski, S., \& Davis, M. 1987, ApJ, 313, 59

Dodelson, S., Gates, E. I., \& Turner, M. S. 1996, Science, 274, 69

Douglas, N. G., et al. 2002, PASP, 114, 1234

Douglas, N. G., et al. 2007, ApJ, 664, 257

Dressler, A., Lynden-Bell, D., Burstein, D., Davies, R. L., Faber, S. M. Terlevich, R., \& Wegner, G. 1987, ApJ, 313, 42

Dubinski, J. 1994, ApJ, 431, 617

Dubinski, J., \& Carlberg, R. G. 1991, ApJ, 378, 496

Eddington, A. S. 1915, MNRAS, 76, 37

Efstathiou, G., Ellis, R. S., \& Carter, D. 1982, MNRAS, 201, 975

Eggen, O. J., Lynden-Bell, D., \& Sandage, A. R. 1962, ApJ, 136, 748

Elmegreen, B. G., Bournaud, F., \& Elmegreen, D. M. 2008, ApJ, 688, 67

Emsellem, E., et al. 2004, MNRAS, 352, 721

Emsellem, E., et al. 2007, MNRAS, 379, 401

Erb, D. K., Steidel, C. C., Shapley, A. E., Pettini, M., Reddy, N. A., \& Adelberger, K. L. 2006, ApJ, 646, 107

Escala, A., Larson, R. B., Coppi, P. S., \& Mardones, D. 2004, ApJ, 607, 765

Faber, S. M., et al. 1997, AJ, 114, 1771

Fakhouri, O., \& Ma, C.-P. 2008, MNRAS, 386, 577

Fall, S. M. 1979, Nature, 281, 200

Fall, S. M., \& Zhang, Q. 2001, ApJ, 561, 751

Farouki, R. T., \& Shapiro, S. L. 1982, ApJ, 259, 103

Ferrarese, L., \& Merritt, D. 2000, ApJ, 539, L9

Foster, C., Proctor, R. N., Forbes, D. A., Spolaor, M., Hopkins, P. F., \& Brodie, J. P. 2009, MNRAS, 400, 2135

Franx, M., \& Illingworth, G. D. 1988, ApJ, 327, L55

Franx, M., Illingworth, G., \& de Zeeuw, T. 1991, ApJ, 383, 112

Fridman, T., \& Merritt, D. 1997, AJ, 114, 1479

Fulton, E. E., \& Barnes, J. E. 2001, MNRAS, 321, 507

Gadotti, D. A. 2009, MNRAS, 393, 1531

Gebhardt, K., et al. 2000, ApJ, 539, L13

Genel, S., Genzel, R., Bouche, N., Naab, T., \& Sternberg, A. 2009a, ApJ, 701, 2002

Genel, S., et al. 2009b, ApJ, 688, 789

Genzel, R., et al. 2008, ApJ, 687, 59

Gerhard, O. E. 1981, MNRAS, 197, 179

Gerhard, O. E. 1983, MNRAS, 203, 19

Gerhard, O. E. 1993, MNRAS, 265, 213

Gerhard, O. E., \& Binney, J. 1985, MNRAS, 216, 467

Gnedin, O. Y., Kravtsov, A. V., Klypin, A. A., \& Nagai, D. 2004, ApJ, 616, 16

Gomez, F. A., \& Helmi, A. 2010, MNRAS, 401, 2285

Gonzalez-Garcia, A. C., Balcells, M., \& Olshevsky, V. S. 2006, MNRAS, 372 , 78

Gonzalez-Garcia, A. C., Onorbe, J., Dominguez-Tenreiro, R., \& GomezFlechoso, M. A. 2009, A\&A, 497, 35

Gonzalez-Garcia, A. C., \& van Albada, T. S. 2003, MNRAS, 342, 36

Goodman, J., \& Schwarzschild, M. 1981, ApJ, 245, 1087

Gultekin, K., et al. 2009, ApJ, 698, 198

Heiligman, G., \& Schwarzschild, M. 1979, ApJ, 233, 872

Henon, M., \& Heiles, C. 1964, AJ, 69, 73

Hernquist, L. 1989, Nature, 340, 687

Hernquist, L. 1990, ApJ, 356, 359

Hernquist, L. 1992, ApJ, 400, 460

Hernquist, L. 1993, ApJ, 409, 548

Hernquist, L., \& Barnes, J. E. 1991, Nature, 354, 210

Hernquist, L., \& Ostriker, J. P. 1992, ApJ, 386, 375

Hernquist, L., \& Spergel, D. N. 1992, ApJ, 399, L117 
Hernquist, L., Spergel, D. N., \& Heyl, J. S. 1993, ApJ, 416, 415

Hill, G. J., MacQueen, P. J., Palunas, P., Kelz, A., Roth, M. M., Gebhardt, K., \& Grupp, F. 2006, New Astron., 50, 378

Hoffman, L. 2007, PhD thesis, Harvard Univ.

Hoffman, L., Cox, T. J., Dutta, S., \& Hernquist, L. 2009, ApJ, 705, 920

Holley-Bockelmann, K., Mihos, J. C., Sigurdsson, S., \& Hernquist, L. 2001, ApJ, 549, 862

Holley-Bockelmann, K., Mihos, J. C., Sigurdsson, S., Hernquist, L., \& Norman, C. 2002, ApJ, 567, 817

Hopkins, P. F., Cox, T. J., \& Hernquist, L. 2008a, ApJ, 689, 17

Hopkins, P. F., Cox, T. J., Keres, D., \& Hernquist, L. 2008b, ApJS, 175, 390

Hopkins, P. F., Cox, T. J., Dutta, S. N., Hernquist, L., Kormendy, J., \& Lauer, T. R. 2009a, ApJS, 181, 135

Hopkins, P. F., Cox, T. J., Younger, J., \& Hernquist, L. 2009b, ApJ, 691, 1168

Hopkins, P. F., Hernquist, L., Cox, T. J., Di Matteo, T., Robertson, B., \& Springel, V. 2006, ApJS, 163, 1

Hopkins, P. F., Hernquist, L., Cox, T. J., Dutta, S. N., \& Rothberg, B. 2008c, ApJ, 679,156

Hopkins, P. F., et al. 2009c, MNRAS, 397, 802

Hopkins, P. F., et al. 2010, ApJ, 715, 202

Hoyle, F., \& Lyttleton, R. A. 1939, Math. Proc. Camb. Phil. Soc., 35, 405

Huss, A., Jain, B., \& Steinmetz, M. 1999, MNRAS, 308, 1011

Illingworth, G. 1977, ApJ, 218, L43

Jedrzejewski, R. I., \& Schechter, P. L. 1988, ApJ, 330, L87

Jeong, H., Yi, S. K., Bureau, M., Krajnovic, D., \& Davies, R. L. 2008, in IAU Symp. 245, Formation and Evolution of Galaxy Bulges, ed. M. Bureau, E. Athanassoula, \& B. Barbuy (Cambridge: Cambridge Univ. Press), 193

Jesseit, R., Cappellari, M., Naab, T., Emsellem, E., \& Burkert, A. 2009, MNRAS, 397, 1202

Jesseit, R., Naab, T., \& Burkert, A. 2005, MNRAS, 360, 1185

Jesseit, R., Naab, T., \& Burkert, A. 2008, arXiv:0808.0570

Jesseit, R., Naab, T., Peletier, R. F., \& Burkert, A. 2007, MNRAS, 376, 997

Johansson, P. H., Naab, T., \& Burkert, A. 2009, ApJ, 690, 802

Kalapotharakos, C., Voglis, N., \& Contopoulos, G. 2004, A\&A, 428, 905

Kandrup, H. E., \& Siopis, C. 2003, MNRAS, 345, 727

Katz, N., Weinberg, D. H., \& Hernquist, L. 1996, ApJS, 105, 19

Kauffmann, G. 1996, MNRAS, 281, 487

Kauffmann, G., Colberg, J. M., Diaferio, A., \& White, S. D. M. 1999, MNRAS, 303,188

Kazantzidis, S., Kravtsov, A. V., Zentner, A. R., Allgood, B., Nagai, D., \& Moore, B. 2004, ApJ, 611, L73

Kennicutt, R. C. 1998, ApJ, 498, 541

Keres, D., \& Hernquist, L. 2009, ApJ, 700, L1

Kormendy, J., \& Bender, R. 1996, ApJ, 464, 119

Kormendy, J., Fisher, D. B., Cornell, M. E., \& Bender, R. 2009, ApJS, 182, 216

Krajnovic, D., et al. 2008, MNRAS, 388, 1537

Lacey, C., \& Cole, S. 1993, MNRAS, 262, 627

Lacey, C., \& Cole, S. 1994, MNRAS, 271, 676

Lauer, T. R., et al. 2005, AJ, 129, 2138

Lauer, T. R., et al. 2007, ApJ, 664, 226

Lutz, D. 1991, A\&A, 245, 31

Lynden-Bell, D. 1967, MNRAS, 136, 101

MacMillan, J. D., Widrow, L. M., \& Henriksen, R. N. 2006, ApJ, 653, 43

Maller, A. H, Prochaska, J. X., Somerville, R. S., \& Primack, J. R. 2001, MNRAS, 326, 1475

Manrique, A., \& Salvador-Sole, E. 1996, ApJ, 467, 504

Martig, M., \& Bournaud, F. 2010, ApJ, 714, L275

McGlynn, T. A. 1984, ApJ, 281, 13

McGlynn, T. A. 1990, ApJ, 348, 515

Merritt, D., \& Fridman, T. 1996, ApJ, 460, 136

Merritt, D., \& Quinlan, G. D. 1998, ApJ, 498, 625

Merritt, D., \& Valluri, M. 1996, ApJ, 471, 82

Merritt, D., \& Valluri, M. 1997, Ann. New York Acad. Sci., 848, 48

Merritt, D., \& Valluri, M. 1999, AJ, 118, 1177

Mihos, J. C., \& Hernquist, L. 1994a, ApJ, 437, L47

Mihos, J. C., \& Hernquist, L. 1994b, ApJ, 431, L9

Mihos, J. C., \& Hernquist, L. 1996, ApJ, 464, 641

Miller, R. H. 1978, ApJ, 223, 122

Miller, R. H., \& Smith, B. F. 1979, ApJ, 227, 407

Miller, R. H., \& Smith, B. F. 1980, ApJ, 235, 793

Murphy, J., Gebhardt, K., Adams, J. J., \& Blanc, G. 2009, BAAS, 41, 233

Naab, T., \& Burkert, A. 2001, ApJ, 555, 91

Naab, T., \& Burkert, A. 2003, ApJ, 597, 893

Naab, T., Burkert, A., \& Hernquist, L. 1999, ApJ, 523, 133

Naab, T., Jesseit, R., \& Burkert, A. 2006a, MNRAS, 372, 839

Naab, T., Johansson, P. H., \& Ostriker, J. P. 2009, ApJ, 699, L178

Naab, T., Johansson, P. H., Ostriker, J. P., \& Efstathiou, G. 2007, ApJ, 658, 71
Naab, T., Khochfar, S., \& Burkert, A. 2006b, ApJ, 636, L81

Naab, T., \& Trujillo, I. 2006, MNRAS, 369, 625

Nantais, J. B., \& Huchra, J. P. 2009, AJ, submitted (arXiv:0912.2119)

Napolitano, N. R., et al. 2009, MNRAS, 393, 329

Navarro, J. F., Frenk, C. S., \& White, S. D. M. 1997, ApJ, 490, 493

Navarro, J. F., et al. 2010, MNRAS, 402, 21

Negroponte, J., \& White, S. D. M. 1983, MNRAS, 205, 1009

Nieto, J.-L., Bender, R., \& Surma, P. 1991, A\&A, 244, 37

Norman, C. A., May, A., \& van Albada, T. S. 1985, ApJ, 296, 20

Norman, C., \& Silk, J. 1983, ApJ, 266, 502

Norris, M. A., et al. 2008, MNRAS, 385, 40

Novak, G. S., Cox, T. J., Primack, J. R., Jonsson, P., \& Dekel, A. 2006, ApJ, 646, L9

Ostriker, J. P., \& Peebles, P. J. E. 1973, ApJ, 186, 467

Ostriker, J. P., \& Steinhardt, P. J. 1995, Nature, 377, 600

Poon, M. Y., \& Merritt, D. 2001, ApJ, 549, 192

Press, W. H., Flannery, B. P., Teukolsky, S. A., \& Vetterling, W. T. 1998, Numerical Recipes in C: The Art of Scientific Computing (New York: Cambridge Univ. Press)

Press, W. H., \& Schechter, P. 1974, ApJ, 187, 425

Proctor, R. N., Forbes, D. A., Brodie, J. P., \& Strader, J. 2008, MNRAS, 385 , 1709

Proctor, R. N., Forbes, D. A., Romanowsky, A. J., Brodie, J. P., Strader, J., Spolaor, M., Mendel, J. T., \& Spitler, L. 2009, MNRAS, 398, 91

Quinlan, G. D., Hernquist, L., \& Sigurdsson, S. 1995, ApJ, 440, 554

Richstone, D. O. 1982, ApJ, 252, 496

Rix, H.-W., \& White, S. D. M. 1990, ApJ, 362, 52

Robertson, B., \& Bullock, J. S. 2008, ApJ, 685, L27

Robertson, B., Bullock, J. S., Cox, T. J., Di Matteo, T., Hernquist, L., Springel, V., \& Yoshida, N. 2006a, ApJ, 645, 986

Robertson, B., Cox, T. J., Hernquist, L., Franx, M., Hopkins, P. F., Martini, P., \& Springel, V. 2006b, ApJ, 641, 21

Robertson, B., Yoshida, N., Springel, V., \& Hernquist, L. 2004, ApJ, 606, 32

Romano-Diaz, E., Shlosman, I., Heller, C., \& Hoffman, Y. 2009, ApJ, 702, 1250

Romanowsky, A. J., Strader, J., Spitler, L. R., Johnson, R., Brodie, J. P., Forbes, D. A., \& Ponman, T. 2009a, AJ, 137, 4956

Romanowsky, A. J., et al. 2003, Science, 301, 1696

Romanowsky, A. J., et al. 2009b, Astro2010: The Astronomy and Astrophysics Decadal Survey, Science White Papers, no. 251 (arXiv:0902.3025)

Saaf, A. F. 1968, ApJ, 154, 483

Schechter, P. L., \& Gunn, J. E. 1979, ApJ, 229, 472

Schmidt, M. 1959, ApJ, 129, 243

Schuberth, Y., Richtler, T., Hilker, M., Dirsch, B., Bassino, L. P., Romanowsky, A. J., \& Infante, L. 2010, A\&A, 513, 52

Schwarzschild, M. 1979, ApJ, 232, 236

Schwarzschild, M. 1993, ApJ, 409, 563

Scorza, C., \& Bender, R. 1995, A\&A, 293, 20

Shapiro, K. L., et al. 2008, ApJ, 682, 231

Sigurdsson, S., Hernquist, L., \& Quinlan, G. D. 1995, ApJ, 446, 75

Siopis, C., \& Kandrup, H. E. 2000, MNRAS, 319, 43

Somerville, R. S., Hopkins, P. F., Cox, T. J., Robertson, B. E., \& Hernquist, L. 2008, MNRAS, 391, 481

Somerville, R. S., \& Primack, J. R. 1999, MNRAS, 310, 1087

Spergel, D. N., \& Hernquist, L. 1992, ApJ, 397, L75

Spergel, D. N., et al. 2007, ApJS, 170, 377

Springel, V. 2000, MNRAS, 312, 859

Springel, V. 2005, MNRAS, 364, 1105

Springel, V., Di Matteo, T., \& Hernquist, L. 2005a, MNRAS, 361, 776

Springel, V., \& Hernquist, L. 2002, MNRAS, 333, 649

Springel, V., \& Hernquist, L. 2003, MNRAS, 339, 289

Springel, V., et al. 2005b, Nature, 435, 629

Springel, V., Yoshida, N., \& White, S. D. M. 2001, New Astron., 6, 79

Sridhar, S., \& Touma, J. 1996, MNRAS, 279, 1263

Stäckel, P. 1890, Math. Ann., 35, 91

Statler, T. S. 1987, ApJ, 321, 113

Statler, T. S. 1994a, ApJ, 425, 458

Statler, T. S. 1994b, ApJ, 425, 500

Statler, T. S., Emsellem, E., Peletier, R. F., \& Bacon, R. 2004, MNRAS, 353, 1

Stewart, K. R., Bullock, J. S., Barton, E. J., \& Wechsler, R. H. 2009a, ApJ, 702, 1005

Stewart, K. L., Bullock, J. S., Wechsler, R. H., \& Maller, A. H. 2009b, ApJ, 702, 307

Stewart, K. L., Bullock, J. S., Wechsler, R. H., Maller, A. H., \& Zentner, A. R. 2008, ApJ, 683, 597

Syer, D., \& Tremaine, S. 1996, MNRAS, 282, 223

Syer, D., \& White, S. D. M. 1998, MNRAS, 293, 337 
Thilker, D. A., et al. 2007, ApJS, 173, 538

Thomas, J., et al. 2009, MNRAS, 393, 641

Toomre, A. 1977, in Evolution of Galaxies and Stellar Populations, ed. B. M.

Tinsley \& R. B. Larson (New Haven, CT: Yale Univ. Obs.), 401

Toomre, A., \& Toomre, J. 1972, ApJ, 178, 623

Tremaine, S., \& Yu, Q. 2000, MNRAS, 319, 1

Valluri, M., Debattista, V. P., Quinn, T., \& Moore, B. 2010, MNRAS, 403, 525

Valluri, M., \& Merritt, D. 1998, ApJ, 506, 686

Valluri, M., Vass, I. M., Kazantzidis, S., Kravtsov, A. V., \& Bohn, C. L. 2007, ApJ, 658, 731

van Albada, T. S. 1982, MNRAS, 201, 939

van de Ven, G., de Zeeuw, P. T., \& van den Bosch, R. C. E. 2008, MNRAS, 385 , 614

van den Bosch, R. C. E. 2008, PhD thesis, Leiden Obs., Leiden Univ.

van den Bosch, R. C. E., \& van de Ven, G. 2008, (arXiv:0811.3474) van den Bosch, R. C. E., van de Ven, G., Verolme, E. K., Cappellari, M., \& de Zeeuw, P. T. 2008, MNRAS, 385, 647

van der Marel, R. P., \& Franx, M. 1993, ApJ, 407, 525

van Dokkum, P. G. 2005, AJ, 130, 2647

Villalobos, A., Kazantzidis, S., \& Helmi, A. 2009, ApJ, submitted, arXiv:0912.2250

Wagner, S. J., Bender, R., \& Moellenhoff, C. 1988, A\&A, 195, 5

Weijmans, A.-M., et al. 2009, MNRAS, 398, 561

Weil, M. L., \& Hernquist, L. 1994, ApJ, 431, L79

Weil, M. L., \& Hernquist, L. 1995, ApJ, 460, 101

White, S. D. M. 1978, MNRAS, 184, 185

White, S. D. M. 1980, MNRAS, 191, 1

White, S. D. M. 1983, in Internal Kinematics and Dynamics of Galaxies, ed. E. Athanassoula (Dordrecht: D. Reidel), 337

White, S. D. M., \& Rees, M. J. 1978, MNRAS, 183, 341 